ARTICLE OPEN

\title{
NFAT inhibitor 11R-VIVIT ameliorates mouse renal fibrosis after ischemia-reperfusion-induced acute kidney injury
}

\author{
Zhi-yong Xie ${ }^{1,2}$, Wei Dong ${ }^{2}$, Li Zhang ${ }^{2}$, Meng-jie Wang ${ }^{2,3}$, Zhen-meng Xiao ${ }^{2,3}$, Yu-hua Zhang ${ }^{1,2}$, Wan-xin Shi ${ }^{1,2}$, Ying Huang ${ }^{1,2}$, \\ Yan Yang ${ }^{1,2}$, Cui-li Li ${ }^{2,3}$, Lei Fu' ${ }^{2}$, Xing-chen Zhao ${ }^{2}$, Rui-zhao $\mathrm{Li}^{2}$, Zhi-lian Li ${ }^{2}$, Yuan-han Chen ${ }^{2}$, Zhi-ming Ye ${ }^{2}$, Shuang-xin Liu ${ }^{2}$, \\ Zheng Dong ${ }^{4,5}$ and Xin-ling Liang ${ }^{2,1,3}$
}

\begin{abstract}
Acute kidney injury (AKI) with maladaptive tubular repair leads to renal fibrosis and progresses to chronic kidney disease (CKD). At present, there is no curative drug to interrupt AKI-to-CKD progression. The nuclear factor of the activated T cell (NFAT) family was initially identified as a transcription factor expressed in most immune cells and involved in the transcription of cytokine genes and other genes critical for the immune response. NFAT2 is also expressed in renal tubular epithelial cells (RTECs) and podocytes and plays an important regulatory role in the kidney. In this study, we investigated the renoprotective effect of 11R-VIVIT, a peptide inhibitor of NFAT, on renal fibrosis in the AKI-to-CKD transition and the underlying mechanisms. We first examined human renal biopsy tissues and found that the expression of NFAT2 was significantly increased in RTECs in patients with severe renal fibrosis. We then established a mouse model of AKI-to-CKD transition using bilateral ischemia-reperfusion injury (Bi-IRI). The mice were treated with 11R-VIVIT (5 mg/kg, i.p.) on Days 1, 3, 10, 17 and 24 after Bi-IRI. We showed that the expression of NFAT2 was markedly increased in RTECs in the AKI-to-CKD transition. 11R-VIVIT administration significantly inhibited the nuclear translocation of NFAT2 in RTECs, decreased the levels of serum creatinine and blood urea nitrogen, and attenuated renal tubulointerstitial fibrosis but had no toxic side effects on the heart and liver. In addition, we showed that 11R-VIVIT administration alleviated RTEC apoptosis after BiIRI. Consistently, preapplication of 11R-VIVIT (100 nM) and transfection with NFAT2-targeted siRNA markedly suppressed TGF $\beta$ induced HK-2 cell apoptosis in vitro. In conclusion, 11R-VIVIT administration inhibits IRI-induced NFAT2 activation and prevents AKIto-CKD progression. Inhibiting NFAT2 may be a promising new therapeutic strategy for preventing renal fibrosis after IR-AKI.
\end{abstract}

Keywords: acute kidney injury; chronic kidney disease; NFAT2; 11R-VIVIT; renal fibrosis; apoptosis; renal tubular epithelial cells; HK-2 cells

Acta Pharmacologica Sinica (2022) 43:2081-2093; https://doi.org/10.1038/s41401-021-00833-y

\section{INTRODUCTION}

Acute kidney injury (AKI) is a common clinical syndrome and is associated with significant morbidity and mortality. Although the majority of patients may recover from AKI, 29.4\% of AKI patients progress to chronic kidney injury (CKD) stage 3 or higher within the subsequent one year [1]. Individuals with AKI have a 4.81-fold increased risk of end-stage kidney disease (ESKD), resulting in a substantial economic burden on public health [2].

Progressive renal fibrosis is the final common and devastating pathway for kidney diseases, including the AKI-to-CKD transition, which is characterized by increased extracellular matrix components and inflammatory cell infiltration [3]. The AKI-to-CKD transition involves multiple signalling pathways and pathogenesis and has become an intervention target for clinical transformation and treatment $[4,5]$. Currently, no effective clinical therapy can reverse renal fibrosis and reduce AKI-to-CKD progression. Injured renal tubular epithelial cells (RTECs) are the primary drivers of renal fibrosis, and the maladaptive repair of injured RTECs after AKI leads to residual abnormalities in kidney structure and function [6]. RTEC apoptosis could result in the occurrence and progression of renal fibrosis by generating proinflammatory and profibrotic paracrine factors [7]. Excessive apoptotic RTECs can activate macrophages and fibroblasts, which release various cytokines and accelerate tubular atrophy and renal interstitial fibrosis, eventually leading to CKD $[7,8]$. Thus, inhibiting RTEC apoptosis might be a potential therapeutic strategy for the AKI-to-CKD transition [9, 10].

The nuclear factor of the activated T cell (NFAT) family was initially identified as a transcription factor and includes five members. Four of these proteins (NFAT1, NFAT2, NFAT3, NFAT4) are regulated by calcium signalling, while NFAT5 is regulated by osmotic stress [11]. The $\mathrm{Ca}^{2+}$-activated phosphatase calcineurin (CaN) dephosphorylates NFATs, promoting NFAT nuclear translocation and activation. NFAT family members are expressed in most immune system cells and are involved in the transcription of

\footnotetext{
${ }^{1}$ The Second School of Clinical Medicine, Southern Medical University, Guangzhou 510515, China; ${ }^{2}$ Division of Nephrology, Guangdong Provincial People's Hospital, Guangdong Academy of Medical Sciences, Guangzhou 510080, China; ${ }^{3}$ School of Medicine, South China University of Technology, Guangzhou 510006, China; ${ }^{4}$ Department of Cellular Biology and Anatomy, Medical College of Georgia at Augusta University, Augusta, GA, USA and ${ }^{5}$ Department of Medical Research, Charlie Norwood Veterans Affairs Medical Center, Augusta, GA, USA

Correspondence: Xin-ling Liang (xinlingliang_ggh@163.com)

These authors contributed equally: Zhi-yong Xie, Wei Dong
}

Received: 8 July 2021 Accepted: 23 November 2021

Published online: 22 December 2021 
cytokine genes and other genes that are critical for the immune response [12]. Moreover, previous work has shown that NFAT2 could also play an important regulatory role in the kidney and is expressed in nonimmune cells, including RTECs and podocytes [13-16]. Recently, substantial evidence suggests that NFAT2 is involved in the apoptosis of several cell types [17, 18]. Our previous studies demonstrated that NFAT2 mediates highglucose-induced podocytes apoptosis $[15,16]$. However, whether NFAT2 is involved in RTEC apoptosis during renal fibrosis has not been investigated. Moreover, previous studies have suggested that NFAT2 promotes the fibrotic process in several organs $[19,20]$. To date, no studies have investigated the effects of NFAT2 on renal fibrosis in AKI-to-CKD progression.

$11 \mathrm{R}$-VIVIT is a cell-permeable peptide inhibitor of NFAT that can directly inhibit the dephosphorylation of NFAT without affecting the activity of $\mathrm{CaN}$ phosphatase or disrupting other CaNdependent pathways [21, 22]. Moreover, the adverse effects observed in response to traditional $\mathrm{CaN}$ inhibitors (CNIs), cyclosporine, and tacrolimus, including the progressive loss of renal function, hypertension, neurotoxicity, and an increased risk of malignancy, have not been observed in response to 11R-VIVIT [21]. At present, 11R-VIVIT has been shown to attenuate albuminuria and alleviate podocyte injury in diabetic kidney disease $[15,16]$. However, the effect of $11 \mathrm{R}$-VIVIT on renal fibrosis in the AKI-to-CKD transition has not been investigated. In the current study, we investigated whether $11 \mathrm{R}$-VIVIT has renoprotective effects on subsequent renal fibrosis after ischemiareperfusion-induced acute kidney injury (IR-AKI).

\section{MATERIALS AND METHODS}

Patients

Renal biopsy tissues from nine patients with IgA nephropathy (IgAN) accompanied by severe renal fibrosis $(n=3)$, patients with IgAN with mild renal fibrosis $(n=3)$ and adjacent normal tissues from renal cell carcinoma patients $(n=3)$ were obtained after the patients signed written informed consent. Severe renal fibrosis was defined as $>25 \%$ tubular atrophy/interstitial fibrosis, while mild renal fibrosis was defined as $<25 \%$ tubular atrophy/interstitial fibrosis. The clinical parameters of the nine patients are provided in Supplementary Table 1. The expression of NFAT1, NFAT2, NFAT3, and NFAT4 was evaluated using the immunofluorescence staining of frozen sections. The study complied with the Declaration of Helsinki and was approved by the Ethics Committee of Guangdong Provincial People's Hospital (No. GDREC2020199A).

\section{Animal experimental protocol}

Six- to eight-week-old male C57BL/6 mice (weight, 20-25 g) were purchased from the Nanjing Biomedical Research Institute of Nanjing University and were housed at the Animal Centre of Guangzhou Forevergen Biosciences Co., Ltd., which was maintained at a temperature of $23 \pm 2{ }^{\circ} \mathrm{C}$ and a humidity of $55 \% \pm 5 \%$. All mice were administered a standard diet and water and were housed in a pathogen-free environment and a 12/12 h light/dark cycle. The animal study was approved by the Animal Ethics and Welfare Committee (No. IACUC-G16034). After a two-week adaptation period, the mice were randomly divided into three groups: shamoperated mice (sham, $n=18$, sham operation was performed without clamping the renal pedicles); ischemia-reperfusion mice (IRI, $n=18$, mice were subjected to bilateral renal pedicle clamping for $25 \mathrm{~min})$; and ischemia-reperfusion mice that were intraperitoneally injected with 11R-VIVIT (IRI + 11R-VIVIT, $n=18$, mice were subjected to $25 \mathrm{~min}$ of ischemia and received $5 \mathrm{mg} / \mathrm{kg} 11 \mathrm{R}$-VIVIT on Days 1, 3, 10,17 , and 24 after the operation). Mice were anaesthetized with an intraperitoneal injection of $60 \mathrm{mg} / \mathrm{kg} 1 \%$ pentobarbital sodium. Bilateral ischemia-reperfusion injury (Bi-IRI) was induced by the placement of an atraumatic microaneurysm clamp (RS-5420, Roboz
Surgical Instrument Co., Inc., Gaithersburg, MD, USA) on the renal pedicle for $25 \mathrm{~min}$ while the mice were warmed with a heating pad $\left(37^{\circ} \mathrm{C}\right)$ throughout the surgery. After surgery, $1 \mathrm{~mL}$ of warm saline was intraperitoneally injected for volume substitution. Fifty-four mice in the sham-operated, IRI and IRI + 11R-VIVIT groups were euthanized on the 2 nd day $(n=18), 14$ th day $(n=18)$, and 28th day $(n=18)$ after the operation to examine serum creatinine (Scr), blood urea nitrogen (BUN), and renal pathology. Blood samples were obtained from the retro-orbital venous plexus, and the left kidneys were harvested and stored in liquid nitrogen until use. The right kidney, heart, and liver were fixed in $4 \%$ paraformaldehyde overnight and embedded in paraffin. Kidney sections $(3 \mu \mathrm{m})$ were stained with Masson's trichrome to evaluate renal fibrosis. Heart and liver sections $(3 \mu \mathrm{m})$ were stained with haematoxylin-eosin (HE) to observe the pathological changes.

Evaluation of renal function and liver function

Renal function was assessed by measuring Scr and BUN. Scr was determined using the QuantiChrom ${ }^{\text {TM }}$ Creatinine Assay Kit (Cat. No. DICA-500, BioAssay Systems, Hayward, CA, USA), and BUN was assessed using the QuantiChrom ${ }^{\mathrm{TM}}$ Urea Assay Kit (Cat. No. DIUR100, BioAssay Systems, Hayward, CA, USA) according to the manufacturer's protocols. Serum aspartate aminotransferase (AST) was detected using the Cloud-Clone Corp Kit (Lot. L210820841, Cloud-Clone Corp, TX, USA).

Cell culture and treatment

HK-2 cells were obtained from the American Tissue Culture Collection (ATCC, Rockville, MD, USA). Cells were cultured in DMEM/F12 (Lot. no. 10092016, Gibco, Thermo Fisher Scientific, Waltham, USA) supplemented with 10\% FBS (Lot. no. 35081005, Gibco, Thermo Fisher Scientific, Waltham, USA), $100 \mathrm{U} / \mathrm{mL}$ penicillin, and $100 \mu \mathrm{g} / \mathrm{mL}$ streptomycin (Lot. no. 127819, Gibco, Thermo Fisher Scientific, Waltham, USA) at $37{ }^{\circ} \mathrm{C}$ with $5 \% \mathrm{CO}_{2}$. Cells were transfected with $50 \mathrm{nM}$ NFAT2 small interfering RNA (siRNA) (No. stB0007239 genOFFTM st-h-NFATC1, RiboBio Co., Ltd. Guangzhou, China) and Lipofectamine 2000 (Lot no. 2125329, Thermo Fisher Scientific, Waltham, USA) for $6 \mathrm{~h}$ or were cultured with $100 \mathrm{nM} 11 \mathrm{R}$-VIVIT (CAS. No. 592517-80-1, MedChemExpress, New Jersey, USA) for $2 \mathrm{~h}$ and then treated with $5 \mathrm{ng} / \mathrm{mL}$ [20] TGF- $\beta 1$ (Lot. AV7321041, R\&D Systems, Minneapolis, MN, USA) for $72 \mathrm{~h}$ in vitro in serum-free DMEM/F12. A scrambled siRNA (No. siN0000001-1-5 siR NC \#1, RiboBio Co., Ltd. Guangzhou, China) was used as a transfection control.

\section{Western blot analysis}

Kidney tissues and HK-2 cells were lysed with RIPA lysis buffer (No. P0013B, Beyotime Institute of Biotechnology, Jiangsu, China) supplemented with the protease inhibitor phenylmethylsulfonyl fluoride (No. P1082, Beyotime Institute of Biotechnology, Jiangsu, China). Lysates were centrifuged for $10 \mathrm{~min}$ at $4{ }^{\circ} \mathrm{C}$, and the supernatants were obtained. Nuclear and cytoplasmic proteins were extracted from cells using a Nuclear and Cytoplasmic Protein Extraction Kit (No. KGP1100, Nanjing KeyGen Biotech. Co. Ltd., Jiangsu, China) according to the manufacturer's instructions. The protein concentrations of the supernatants were determined using a bicinchoninic acid protein assay kit (Lot. no. WF327088, Thermo Fisher Scientific, Waltham, USA). Proteins were subjected to SDS-PAGE on $7.5 \%-12 \%$ gels and transferred to polyvinylidene difluoride membranes (No. IPVH00010, Millipore, Billerica, MA, USA). The membranes were blocked with $5 \%$ nonfat dry milk in Tris-buffered saline-Tween for $1 \mathrm{~h}$ at room temperature and incubated overnight at $4{ }^{\circ} \mathrm{C}$ with anti- $\beta$-actin $(1: 1,000$; cat. no. 8457; Cell Signalling Technology, Danvers, MA, USA), anti-GAPDH (1:5,000; cat. no. AP0063; Bioworld Technology, St. Louis Park, MN, USA), anti-histone H3 (1:1,000; cat. no. AF0863; Affinity Biosciences, USA), anti-NFAT2 (1:1,000; cat. no. ab25916; Abcam, Cambridge, MA, USA), anti-phospho-NFAT2 (Ser 172; 1:1,000; cat. no. AF8293; Affinity Biosciences, USA), anti-fibronectin (1:1,000; 
cat. no. ab2413; Abcam, Cambridge, MA, USA), anti-a-SMA (1:500; cat. no. ab5694; Abcam, Cambridge, MA, USA), anti-caspase-3 (1:1,000; cat. no. 9662; Cell Signalling Technology, Danvers, MA, USA), anti-cleaved caspase-3 (1:1,000; cat. no. 9664; Cell Signalling Technology, Danvers, MA, USA), and anti-Bax (1:1,000; cat. no. 2772; Cell Signalling Technology, Danvers, MA, USA) antibodies. The membranes were then incubated with a horseradish peroxidase-conjugated goat anti-rabbit $(1: 3,000$; cat. no. 7074; Cell Signalling Technology, Danvers, MA, USA) or anti-mouse (1:3,000; cat. no. 7072; Cell Signalling Technology, Danvers, MA, USA) secondary antibody for $1 \mathrm{~h}$ at room temperature. After being washed in TBST, the protein bands were visualized using Pierce ${ }^{\mathrm{TM}}$ ECL Western blotting Substrate (Lot: 201209-86, Thermo Fisher Scientific, Waltham, USA) and detected with Image Quant LAS 500 (GE Healthcare Life Sciences, USA). ImageJ software was used to analyse the band intensity, and the data were standardized to $\beta$ actin, GAPDH or histone H3 (nuclear fractions).

mRNA extraction, reverse transcription, and quantitative polymerase chain reaction ( $R T-q P C R)$

Total RNA was extracted from the kidney using TRIzol ${ }^{\circledR}$ reagent (Lot: 326401, Invitrogen, Thermo Fisher Scientific, Waltham, USA), and reverse transcription (RT) was carried out using a PrimeScript RT Reagent Kit (Cat \#RR047A, Takara Bio Inc., Madison, WI, USA). CDNA was generated and subjected to PCR using Power SYBR Green PCR Master Mix (Cat \#RR820A, Takara Bio Inc., Madison, WI, USA). The data were analysed using the $2^{-\Delta \Delta C q}$ method, and GAPDH was used as the internal control. The primer sequences are shown in Table 1.

Flow cytometric analysis

A fluorescein isothiocyanate (FITC) annexin-V apoptosis detection kit I was purchased from Dojindo Laboratories (AD10, Kumamoto, Kyushu, Japan) and used to determine the level of apoptosis according to the manufacturer's instructions. The medium was removed from the cells, and then the cells were washed three times with cold PBS and digested with $0.25 \%$ EDTA-free trypsin (Lot. no. 15050065, Gibco, Thermo Fisher Scientific, Waltham, USA). After centrifugation for $5 \mathrm{~min}$ at $140 \times g$ at room temperature, the cells were washed twice with cold PBS and resuspended in $1 \times$ binding buffer at a concentration of $1 \times 10^{6}$ cells $/ \mathrm{mL}$. One hundred microlitres of the solution was transferred to a $5 \mathrm{~mL}$ culture tube, and $5 \mu \mathrm{L}$ of FITC Annexin V and PI were each added. The cells were gently vortexed and incubated for $15 \mathrm{~min}$ at room temperature $\left(25^{\circ} \mathrm{C}\right)$ in the dark. Then, $400 \mu \mathrm{L}$ of $1 \times$ binding buffer was added to each tube, and apoptosis was analysed by flow cytometry (CytoFLEX, Beckman Coulter, Indianapolis, USA) within $1 \mathrm{~h}$.

Immunofluorescence staining

Frozen sections were fixed with $4 \%$ paraformaldehyde at room temperature for $15 \mathrm{~min}$, permeabilized with $0.5 \%$ Triton X-100 for 10 min, and incubated with 5\% BSA for $30 \mathrm{~min}$ at room temperature. The frozen sections were incubated with anti-NFAT1 (1:200; cat. no.

\begin{tabular}{|lc|}
\hline Table 1. & Sequences of primers for RT-qPCR. \\
\hline Gene & Primer sequences \\
\hline GAPDH (mouse) & $\begin{array}{l}\text { Forward: 5'-AGGTCGGTGTGAACGGATTTG-3' } \\
\text { Reserve: 5'-TGTAGACCATGTAGTTGAGGTCA-3' }\end{array}$ \\
$\alpha$-SMA (mouse) & $\begin{array}{l}\text { Forward: 5'-CCCAGACATCAGGGAGTAATGG-3' } \\
\text { Reserve: 5'-TCTATCGGATACTTCAGCGTCA-3' }\end{array}$ \\
Fibronectin (mouse) & Forward: 5'-ATGTGGACCCCTCCTGATAGT-3' \\
& Reserve: 5'-GCCAGTGATTTCAGCAAAGG-3' \\
\hline RT-qPCR reverse transcription-quantitative polymerase chain reaction.
\end{tabular}

5861; Cell Signalling Technology, Danvers, MA, USA), anti-NFAT2 (1:50; cat. no. sc-7294; Santa Cruz Biotechnology, Dallas, TX, USA), anti-NFAT3 (1:100; cat. no. PA1-021; Gibco, Thermo Fisher Scientific, Waltham, USA), and anti-NFAT4 (1:50; cat. no. sc-8405; Santa Cruz Biotechnology, Dallas, TX, USA) antibodies overnight at $4{ }^{\circ} \mathrm{C}$. The frozen sections were washed three times in PBS and incubated with Alexa Fluor 488-conjugated goat anti-rabbit (1:500; cat. no. sc-4412; Santa Cruz Biotechnology, Dallas, TX, USA) or goat anti-mouse (1:500; cat. no. sc-4408; Santa Cruz Biotechnology, Dallas, TX, USA) secondary antibodies for $1 \mathrm{~h}$ at room temperature. The sections were counterstained with DAPI to label the nuclei. Images of each frozen section were captured using a confocal microscope (Nikon C2 Confocal Microscope, Nikon), and the mean grey value was analysed using ImageJ software (version 1.52; National Institutes of Health).

\section{TUNEL staining}

TUNEL staining was performed using an in situ Cell Death Detection Kit (LOT 44446200, Roche Diagnostics GmbH, Mannheim, Germany). Frozen sections or cells in 6-well plates were fixed in $4 \%$ paraformaldehyde for $20 \mathrm{~min}$ at room temperature. After being washed with PBS three times, the frozen sections or cells in 6-well plates were blocked with $3 \% \mathrm{H}_{2} \mathrm{O}_{2}$ in methanol for $10 \mathrm{~min}$ at room temperature and then permeabilized with $0.1 \%$ Triton $\mathrm{X}-100$ in $0.1 \%$ sodium citrate for $10 \mathrm{~min}$ at room temperature. Each frozen section or well of cell plates was incubated with $500 \mu \mathrm{L}$ of TUNEL reaction mixture containing $50 \mu \mathrm{L}$ of the enzyme solution and $450 \mu \mathrm{L}$ of the label solution for $60 \mathrm{~min}$ at $37{ }^{\circ} \mathrm{C}$ in humidified and dark conditions. After being washed three times with PBS, the frozen sections or cells in 6-well plates were stained with DAPI for $10 \mathrm{~min}$ at room temperature and placed on coverslips. Six fields were captured for each frozen section ( $n=3$ in each group) using a confocal fluorescence microscope (Nikon C2 Confocal Microscope, Nikon), and positively stained cells were quantified and analysed using ImageJ software (version 1.52; National Institutes of Health).

\section{Statistical analysis}

All data were analysed using SPSS statistical software for Windows, version 23.0 (SPSS, Inc., Chicago, IL, USA). The data were analysed by one-way ANOVA for multiple comparisons, followed by Bonferroni's post hoc analysis. Comparisons between two groups were assessed using Student's $t$ tests. Two-tailed tests were used for all comparisons, and $P<0.05$ was considered statistically significant.

\section{RESULTS}

NFAT2 was markedly increased in human renal tissue with severe fibrosis

It is difficult to obtain human kidney samples during the AKI-toCKD transition because patients diagnosed with AKI seldom undergo renal biopsies. We evaluated the expression of NFAT1, NFAT2, NFAT3 and NFAT4 in frozen sections from three biopsyproven IgAN patients with severe renal fibrosis and three IgAN patients with mild renal fibrosis. Adjacent normal renal tissue from three renal cell carcinoma patients was used as a control. Immunofluorescence analysis showed increased the expression and nuclear localization of NFAT2 in the renal tubules, interstitium, and glomerulus in IgAN patients with severe renal fibrosis compared with IgAN patients with mild renal fibrosis and the controls. However, the expression of NFAT1, NFAT3, and NFAT4 was similar in IgAN patients with various degrees of fibrosis and the controls (Fig. 1a-f).

NFAT2 was activated in the animal model of AKI-to-CKD progression

We used the Bi-IRI model to longitudinally examine CKD progression after AKI. The levels of Scr and BUN were significantly elevated after IRI compared to those of sham-operated mice on 
a

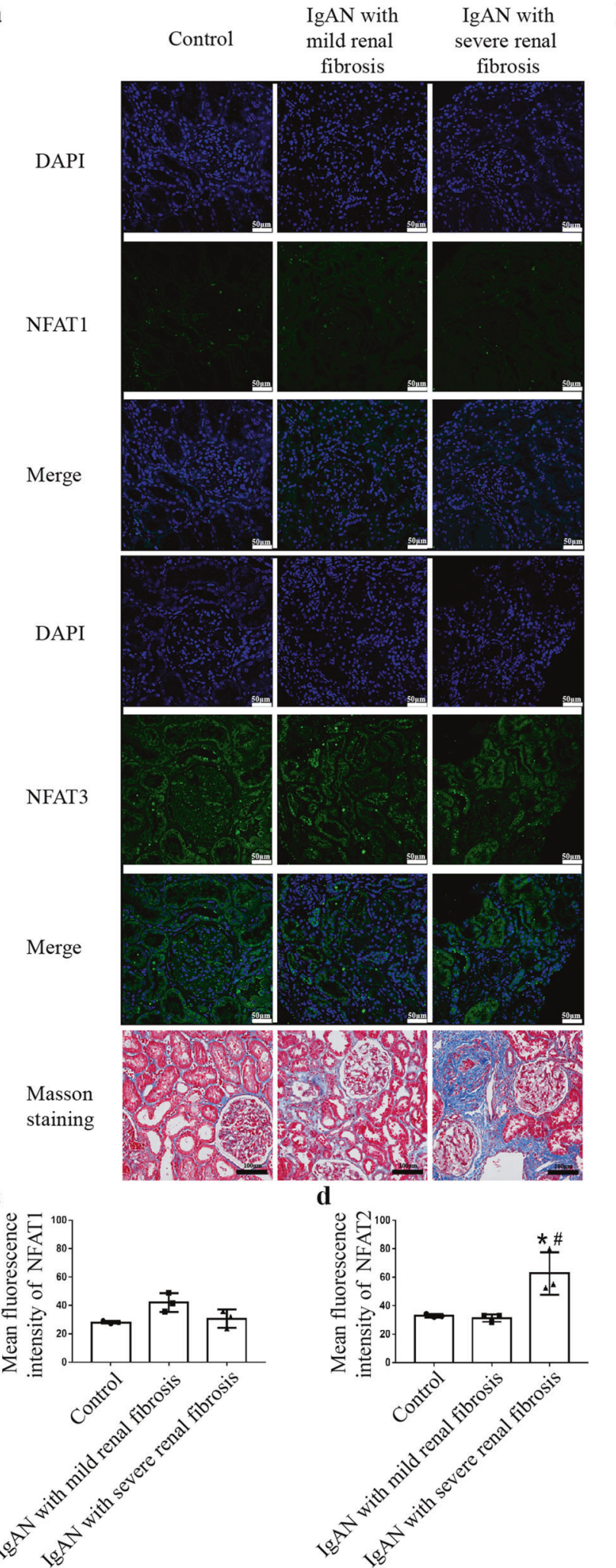

b

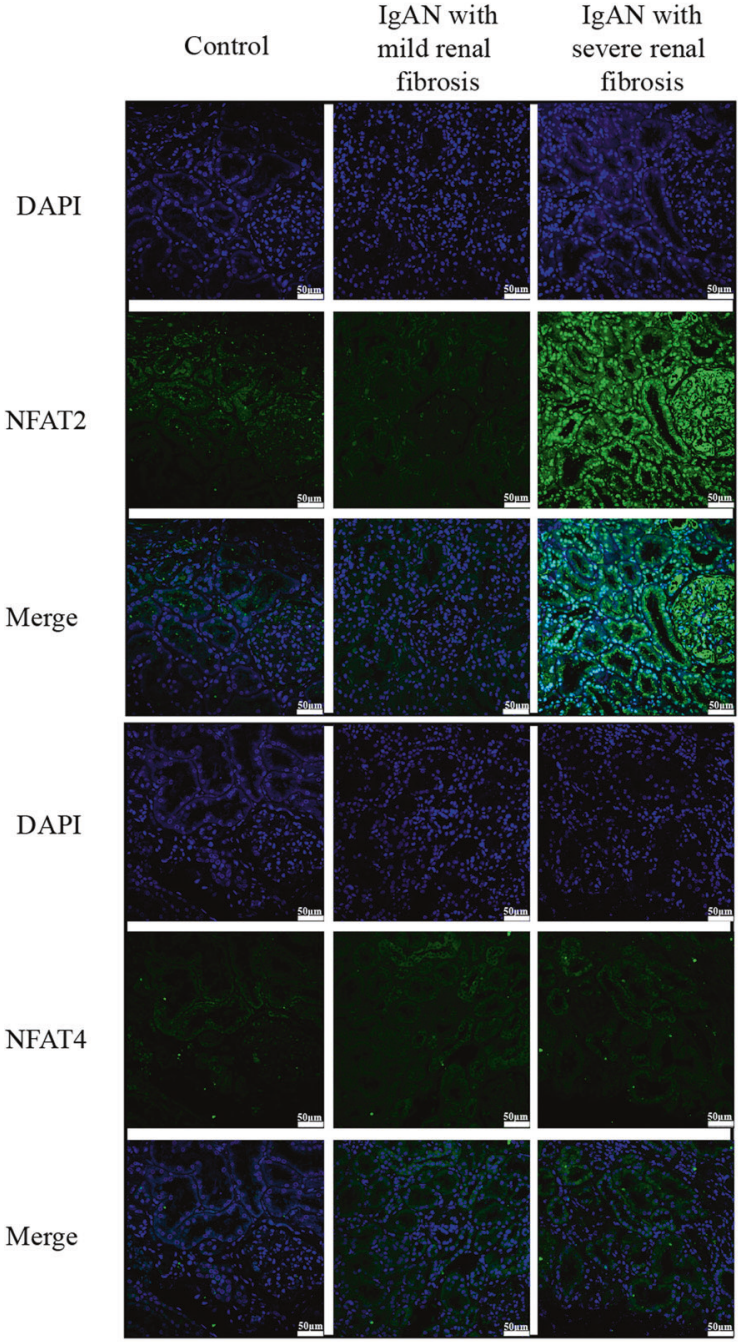

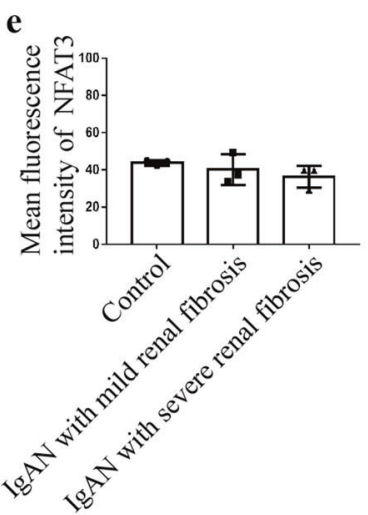

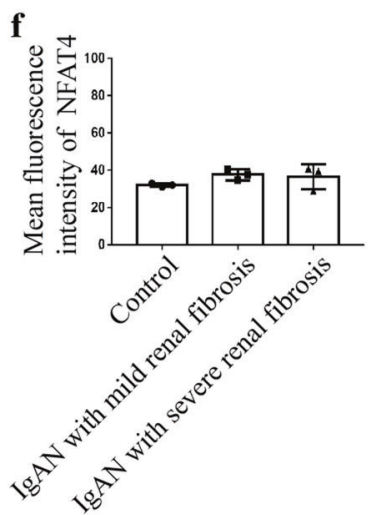

Fig. 1 NFAT2 is markedly increased in human renal tissue with severe fibrosis. Immunostaining for NFAT1, NFAT2, NFAT3 and NFAT4 was performed on tissues from IgAN patients and adjacent normal tissues from renal cell carcinoma patients. Representative confocal images showed higher expression and more nuclear localization of NFAT2 in the renal tubules, interstitium and glomerulus in IgAN patients with severe renal fibrosis $(n=3)$ than in IgAN patients with mild renal fibrosis or in adjacent normal renal tissue from renal cell carcinoma patients $(n=3)$ (Scale bars $=50 \mu \mathrm{m})$. Masson staining was used to assess kidney fibrosis (scale bars $=100 \mu \mathrm{m})$. a Immunostaining for NFAT1 and NFAT3. b Immunostaining for NFAT2 and NFAT4. c-f Quantitative analysis for the immunofluorescence of NFAT1, NFAT2, NFAT3 and NFAT4. * $P<0.05$ vs. Control; ${ }^{\#} P<0.05$ vs. IgAN with mild renal fibrosis. NFAT1 nuclear factor of activated T cells 1 , NFAT2 nuclear factor of activated T cells 2 , NFAT3 nuclear factor of activated T cells 3, NFAT4 nuclear factor of activated T cells 4. 
$\mathbf{a} \rightleftharpoons$

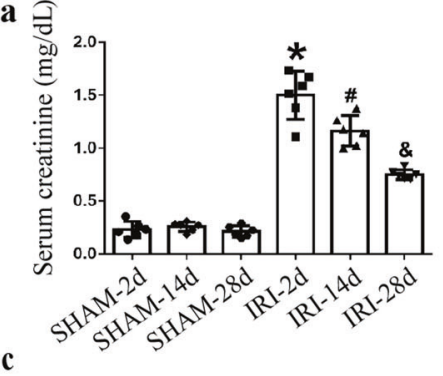

b

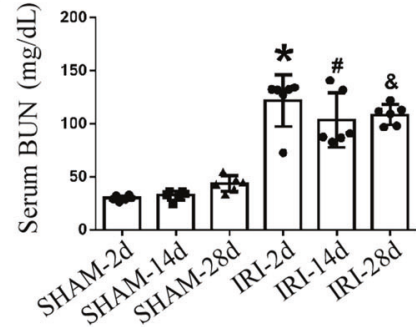

d

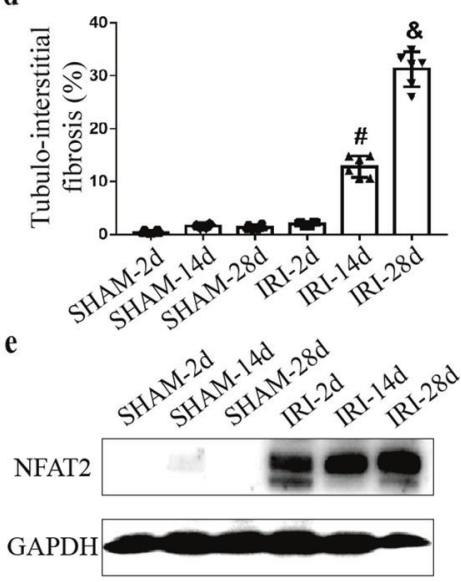

f

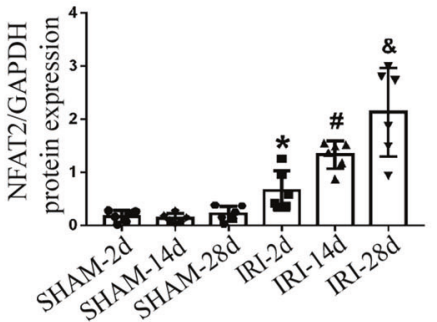

Fig. 2 NFAT2 is increased in the renal tissue of AKI-to-CKD transition animal model. a Scr and (b) serum BUN levels were measured in the sham-operated and IRI models. c Masson staining showed renal histopathology fibrosis. Scale bars $=100 \mu \mathrm{m}$. d Quantification of tubulointerstitial fibrosis. e-f Protein expression of NFAT2 in the kidneys of sham-operated (2d, 14d, 28d) and IRI (2d, 14d, 28d) mice, as determined by Western blotting and densitometric analysis. ${ }^{*} P<0.05$ vs. SHAM-2d, ${ }^{\#} P<0.05$ vs. SHAM-14d, ${ }^{\circledR} P<0.05$ vs. SHAM-28d. NFAT2 nuclear factor of activated T cells 2, AKI acute kidney injury, CKD chronic kidney disease, Scr serum creatinine, BUN blood urea nitrogen, IRI ischemia-reperfusion injury, NFAT1 nuclear factor of activated T cells 1, NFAT3 nuclear factor of activated T cells 3, NFAT4 nuclear factor of activated T cells 4.

the 2nd day. Scr and BUN decreased on the 14th and 28th days compared with the 2 nd day but did not completely return to baseline levels after IRI (Fig. 2a and b). Masson staining showed that the degree of renal interstitial fibrosis was more severe on the 14th and 28th days than in sham-operated mice at the corresponding times (Fig. $2 \mathrm{c}$ and d). Western blot analysis revealed the higher expression of NFAT2 in the IRI group than in the sham group on the 2nd, 14th, and 28th days, and the expression of NFAT2 in the IRI-28d group was higher than that in the IRI-2d and IRI-14d groups (Fig. 2e and f). Immunofluorescence analysis showed the increased expression and nuclear localization of NFAT2 in RTECs in the IRI-2d, 14d, and 28d groups compared with the corresponding sham-operated groups, while the glomerulus did not show elevated NFAT2 expression. Consistent with our human kidney biopsy results, NFAT1, NFAT3, and NFAT4 were not obviously upregulated in RTECs of the AKI-to-CKD animal model in the IRI-2d, 14d, and 28d groups (Fig. 3a-e). Our results indicated that NFAT2 was increased in RTECs in the AKI-to-CKD progression model.

11R-VIVIT attenuated tubulointerstitial fibrosis in the AKI-to-CKD progression model

As there is currently no specific NFAT2 inhibitor, we used the NFAT inhibitor 11R-VIVIT to inhibit NFAT2 activity. Mice were treated with $5 \mathrm{mg} / \mathrm{kg} 11 \mathrm{R}$-VIVIT on Days 1, 3, 10, 17, and 24 after IRI. We examined the inhibitory effect of 11R-VIVIT on the nuclear localization of NFAT2 by immunofluorescence staining. 11R-VIVIT treatment significantly reduced the nuclear localization of NFAT2 in RTECs in the AKI-CKD transition model (Fig. 4a and b). Moreover, Western blot (Fig. 4c and d) analysis showed that the expression of phosphorylated NFAT2 was decreased in the IRI group on the 2nd, 14th, and 28th days and that 11R-VIVIT treatment significantly increased the expression of phosphorylated NFAT2. These results indicated that 11R-VIVIT could inhibit the nuclear localization and dephosphorylation of NFAT2.

11R-VIVIT significantly improved renal function compared with that in the IRI group on the 28th day (Fig. 5a and b). 11R-VIVIT treatment also alleviated IRI-induced interstitial fibrosis (Fig. $5 \mathrm{e}$ and $\mathrm{f}$ ). In addition, RT-qPCR (Fig. $5 \mathrm{c}$ and $\mathrm{d}$ ) and Western blot (Fig. $5 \mathrm{~g}, \mathrm{~h}$, and i) analysis revealed that the expression of the fibrotic markers a-SMA and fibronectin was decreased in the IRI + $11 \mathrm{R}$-VIVIT group on the 28th day. These results revealed that 11RVIVIT could attenuate tubulointerstitial fibrosis in the AKI-to-CKD progression model.

Furthermore, we evaluated the toxic side effects of 11R-VIVIT on other organs, including the heart and liver. HE staining showed that the pathological changes in heart and liver tissue were similar in the sham operation, IRI, and IRI + 11R-VIVIT groups (Supplementary Fig. 1). There was no difference in the level of serum AST between the sham operation, IRI, and IRI + 11R-VIVIT groups (Supplementary Fig. 2). These results indicated that 11R-VIVIT had no toxic side effects on the heart and liver.

11R-VIVIT reduced tubular epithelial cell apoptosis in the AKI-toCKD progression model

TUNEL staining was used to evaluate apoptosis in the AKI-to-CKD progression animal model. The number of TUNEL-positive RTECs was significantly higher in the IRI group than in the shamoperated group on the 2nd, 14th, and 28th days (Fig. 6a and b). Western blot analysis showed that the expression of 
SHAM-2d SHAM-14d SHAM-28d IRI-2d IRI-14d IRI-28d

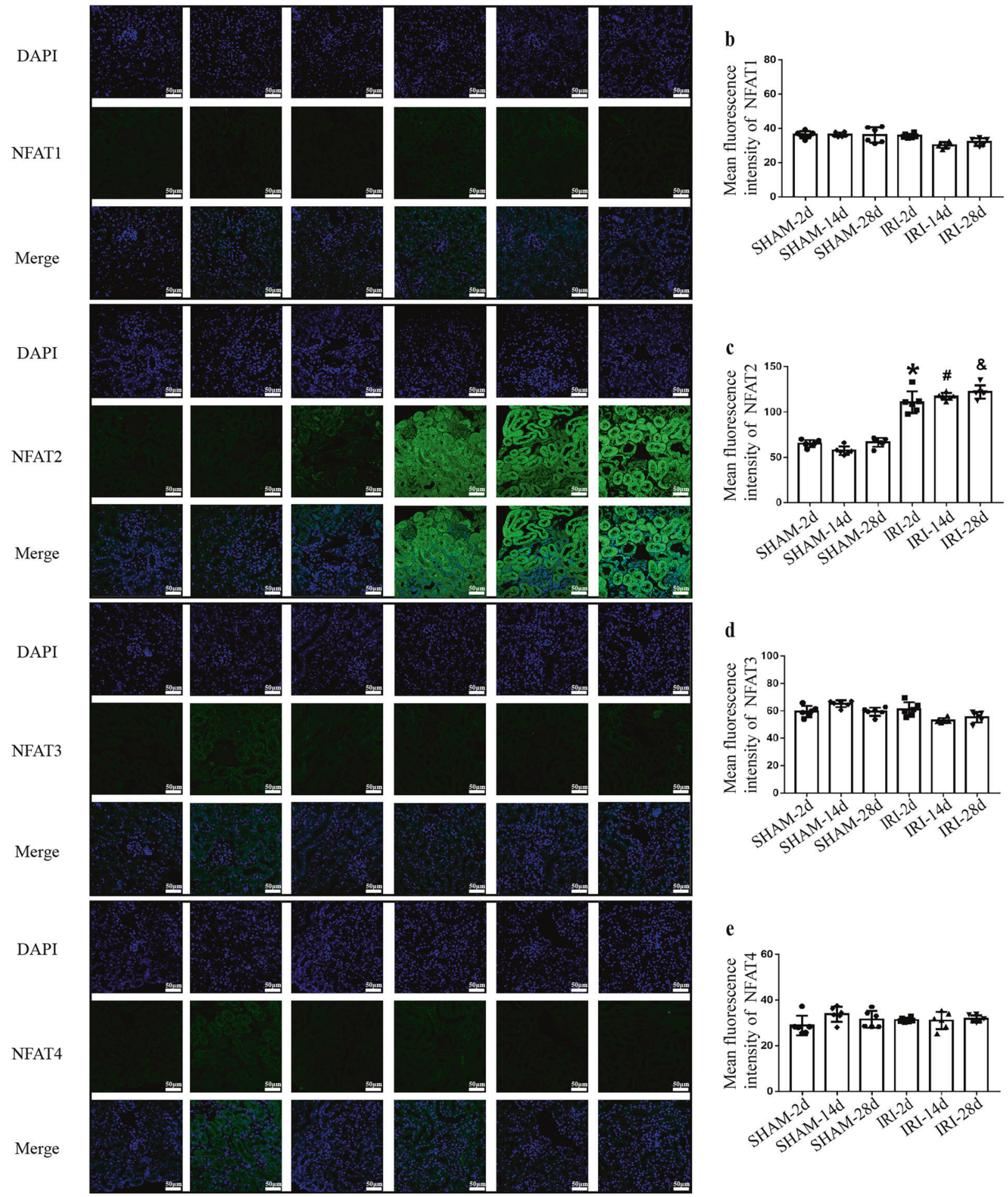

Fig. 3 NFAT2 is activated in the RTECs of AKI-to-CKD transition animal model. a Immunostaining for NFAT1, NFAT2, NFAT3, NFAT4 (green) and DAPI (blue) was performed in frozen renal sections from sham-operated $(2 \mathrm{~d}, 14 \mathrm{~d}, 28 \mathrm{~d})$ and IRI $(2 \mathrm{~d}, 14 \mathrm{~d}, 28 \mathrm{~d}) \mathrm{mice}$. Scale bars $=50 \mu \mathrm{m}$. b-e Quantitative analysis for the immunofluorescence of NFAT1, NFAT2, NFAT3 and NFAT4. ${ }^{*} P<0.05$ vs. SHAM-2d, ${ }^{\#} P<0.05$ vs. SHAM-14d, ${ }^{*} P<$ 0.05 vs. SHAM-28d. NFAT2 nuclear factor of activated T cells 2, RTECs renal tubular epithelial cells, AKI acute kidney injury, CKD chronic kidney disease, NFAT1 nuclear factor of activated T cells 1, NFAT3 nuclear factor of activated T cells 3, NFAT4 nuclear factor of activated T cells 4, IRI ischemia-reperfusion injury. 

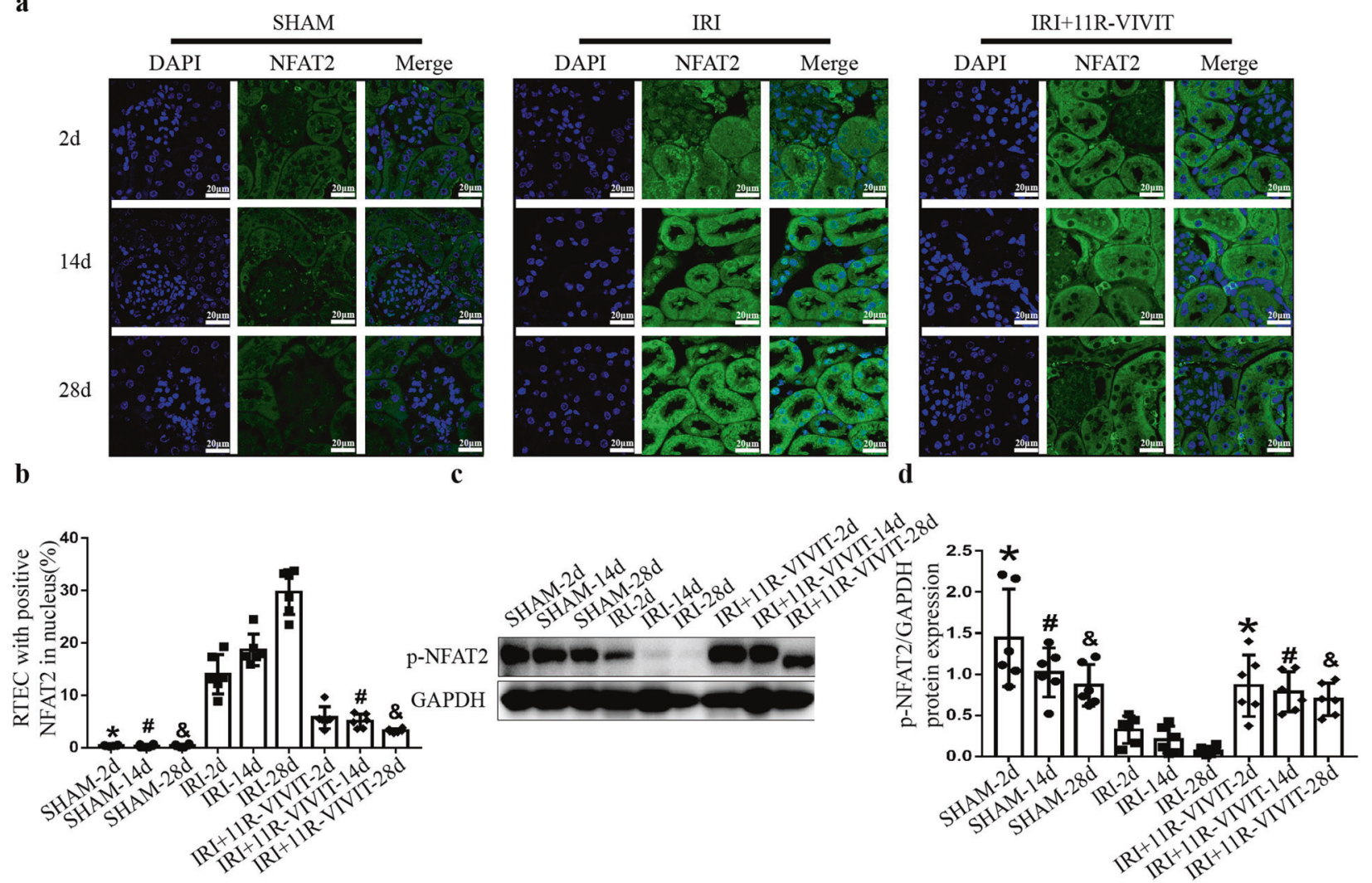

Fig. 4 11R-VIVIT inhibits the nuclear localization and dephosphorylation of NFAT2 in RTECs in an AKI-to-CKD progression model. a Double immunofluorescence staining of NFAT2 (green) and DAPI (blue) and merged images of kidneys from the sham-operated, IRI and IRI $+11 \mathrm{R}$-VIVIT treatment groups. Scale bars $=20 \mu \mathrm{m}$. $\mathbf{b}$ Quantification of the percentage of renal tubular epithelial cells with NFAT2 expression in the nucleus. ${ }^{*} P<0.05$ vs. IRI-2d, ${ }^{\#} P<0.05$ vs. IRI-14d, ${ }^{\&} P<0.05$ vs. IRI-28d. c Protein expression of $p-N F A T 2$ (Ser172) in the sham-operated, IRI and IRI $+11 \mathrm{R}$-VIVIT treatment groups on the 2nd, 14th and 28th day. $\mathbf{d}$ The quantitative results of p-NFAT2 (Ser172) were normalized to GAPDH. NFAT2 nuclear factor of activated T cells 2, RTECs renal tubular epithelial cells, AKI acute kidney injury, CKD chronic kidney disease, IRI ischemia-reperfusion injury, p-NFAT2 phosphorylated nuclear factor of activated T cells 2 .

cleaved-caspase-3, a marker of apoptosis, was significantly increased in the kidney tissues of the IRI group on the 2nd, 14th, and 28th days (Fig. 6c, d, and e). Bax, a proapoptotic member of the $\mathrm{Bcl}-2$ family, was also significantly increased in IRI mice on the 2nd, 14th, and 28th days (Fig. 6f and g). These results indicated that apoptosis was induced in RTECs in the AKI-to-CKD progression model. After 11R-VIVIT intervention, the number of TUNEL-positive RTECs decreased in the IRI + 11R-VIVIT group compared with the IRI group on the 14th and 28th days (Fig. 6a and b). 11R-VIVIT treatment also reduced cleaved-caspase-3 (Fig. $6 c$, d, and e) and Bax (Fig. $6 \mathrm{f}$ and g) expression on the 28th day.

NFAT2-targeted siRNA attenuated TGF $\beta$-induced tubular epithelial cell apoptosis

To further investigate the role of NFAT2 in the AKI-to-CKD transition, we used siRNA to inhibit the expression of NFAT2. siRNA targeting the NFAT2-001 sequence significantly reduced the mRNA and protein levels of NFAT2 (Fig. 7a, b, and c). Transfection with NFAT2-targeted siRNA decreased the elevated expression of a-SMA and fibronectin induced by TGF $\beta$ treatment (Fig. 7d, e, and f). Moreover, flow cytometry showed that NFAT2-targeted siRNA decreased TGF $\beta$-induced tubular epithelial cell apoptosis (Fig. $7 \mathrm{~g}$ and $\mathrm{h}$ ). Western blot analysis suggested that NFAT2-targeted siRNA decreased the expression of cleaved caspase-3 and Bax in HK-2 cells after TGF $\beta$ treatment (Fig. 7i-I). The number of TUNEL-positive RTECs was significantly increased in HK-2 cells after TGF $\beta$ treatment and could be reduced by transfection with
NFAT2-targeted siRNA (Fig. $7 \mathrm{~m}$ and $\mathrm{n}$ ). These data suggested that NFAT2 could mediate tubular epithelial cell apoptosis.

11R-VIVIT attenuated TGF $\beta$-induced tubular epithelial cell apoptosis in vitro

The effect of 11R-VIVIT on NFAT2 activity in HK-2 cells after TGF $\beta$ treatment was assessed in vitro. TGF $\beta$ treatment increased the expression of NFAT2 (Fig. 8a, c). Moreover, it decreased the expression of phosphorylated NFAT2 (Fig. 8b, d) and increased the nuclear localization of NFAT2 in HK-2 cells, and these effects were blocked by the administration of 11R-VIVIT (Fig. 8e-h). 11R-VIVIT also decreased the elevated expression of a-SMA and fibronectin induced by TGF $\beta$ treatment (Fig. $8 \mathrm{i}$, j, and k). Flow cytometry showed that 11R-VIVIT decreased TGF $\beta$-induced tubular epithelial cell apoptosis (Fig. $8 \mathrm{l}$ and $\mathrm{m}$ ). 11R-VIVIT also decreased cleaved caspase- 3 and Bax levels in HK-2 cells after TGF $\beta$ treatment (Fig. $8 n-q)$. The increased number of TUNEL-positive RTECs induced by TGF $\beta$ treatment was reduced by $11 \mathrm{R}$-VIVIT treatment (Fig. $8 \mathrm{r}$ and s). These data suggested that 11R-VIVIT could inhibit TGF- $\beta$-induced tubular epithelial cell apoptosis in vitro.

\section{DISCUSSION}

In this study, we demonstrated that, among the NFAT subtypes, only the expression of NFAT2 varies with the degree of fibrosis. NFAT2 was markedly increased in IgAN patients with severe renal fibrosis, while the expression of NFAT1, NFAT3, and NFAT4 was similar in IgAN patients with various degrees of fibrosis and the 


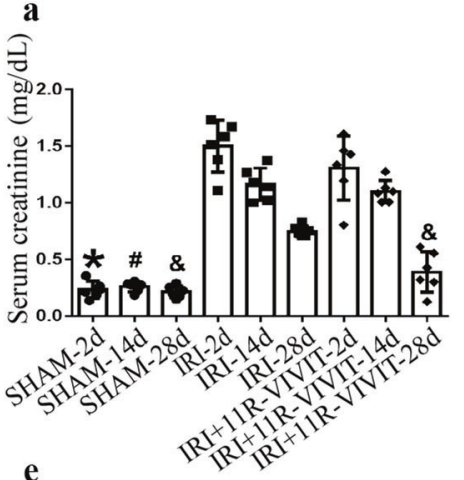

e
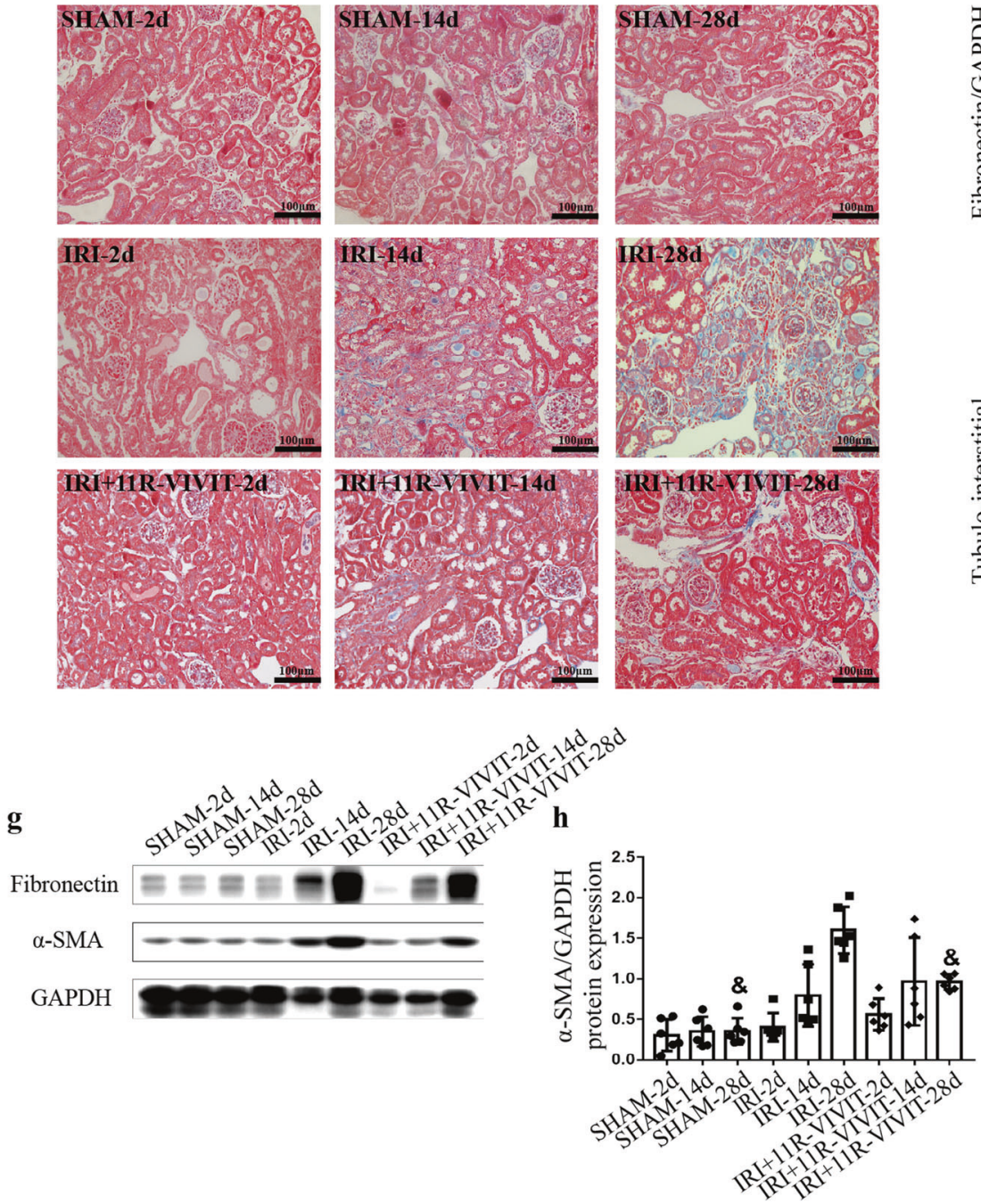
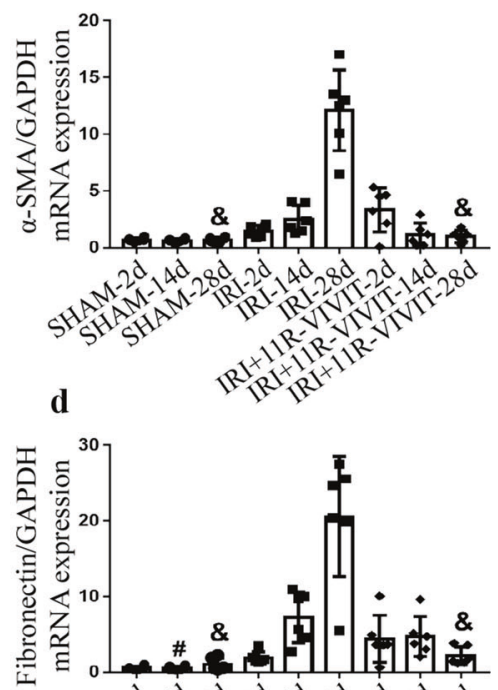

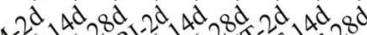
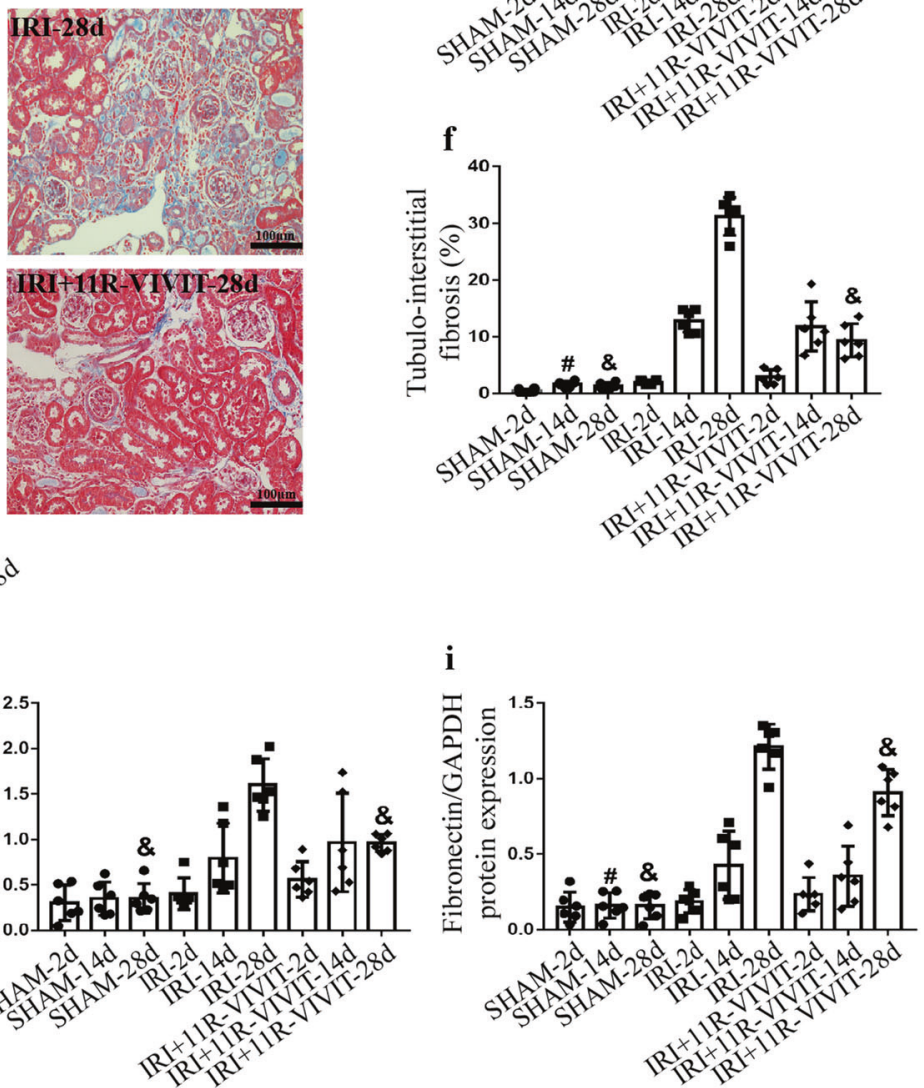

Fig. 5 11R-VIVIT attenuates tubulointerstitial fibrosis in an animal model of AKI-to-CKD progression. a Scr and (b) serum BUN levels were determined in the sham-operated, IRI and IRI + 11R-VIVIT treatment groups on the 2nd, 14th and 28th day. c-d $\alpha$-SMA and fibronectin mRNA expression was measured in each group by RT-qPCR on the 2nd, 14th and 28th day. e Masson staining showed renal histopathological fibrosis in each group on the $2 \mathrm{nd}, 14 \mathrm{th}$ and $28 \mathrm{th}$ day. Scale bars $=100 \mu \mathrm{m}$. $\mathbf{f}$ Quantification of tubulointerstitial fibrosis. $\mathrm{g}$ Protein expression of $\alpha$-SMA and fibronectin in each group on the 2nd, 14th and 28th day. $\mathbf{h}-\mathbf{i}$ The quantitative results of $\alpha$-SMA and fibronectin were normalized to GAPDH. ${ }^{*} P<0.05$ vs. IRI-2d, ${ }^{\#} P<0.05$ vs. IRI-14d, ${ }^{\&} P<0.05$ vs. IRI-28d. AKI acute kidney injury, CKD chronic kidney disease, Scr serum creatinine, BUN blood urea nitrogen, IRI ischemia-reperfusion injury, RT-qPCR reverse transcription-quantitative polymerase chain reaction.

controls. As renal fibrosis is a common consequence of various progressive kidney diseases, including the AKI-to-CKD transition, we further explored the expression of NFAT subtypes in the animal model of AKI-to-CKD progression. Consistently, we found that NFAT2 but not the other NFAT subtypes was significantly upregulated in RTECs, accompanied by increased nuclear accumulation of NFAT2. These data indicated that NFAT2 in RTECs was activated in AKI-to-CKD progression.

Increasing evidence has demonstrated that NFAT2 plays a proapoptotic role through the intrinsic and extrinsic apoptotic 
SHAM

DAPI TUNEL Merge

$2 \mathrm{~d}$

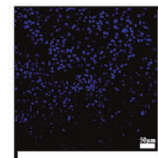

$14 \mathrm{~d}$

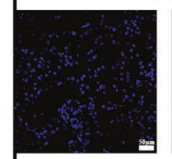

$28 d$
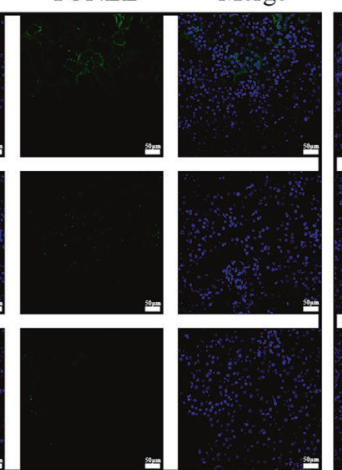
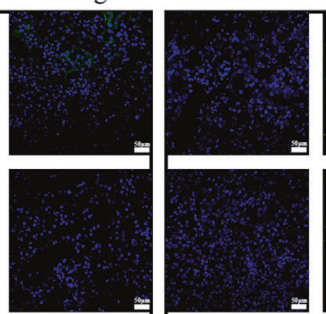

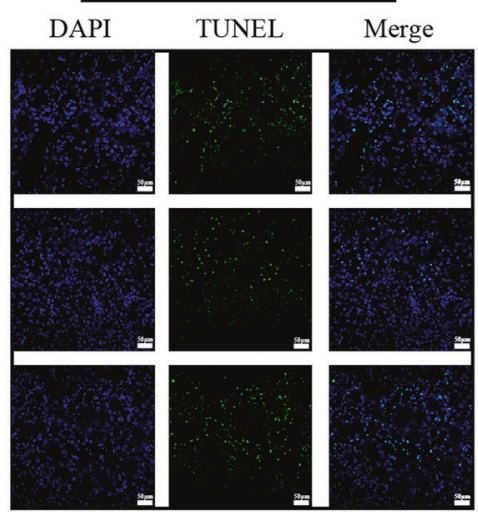

IRI

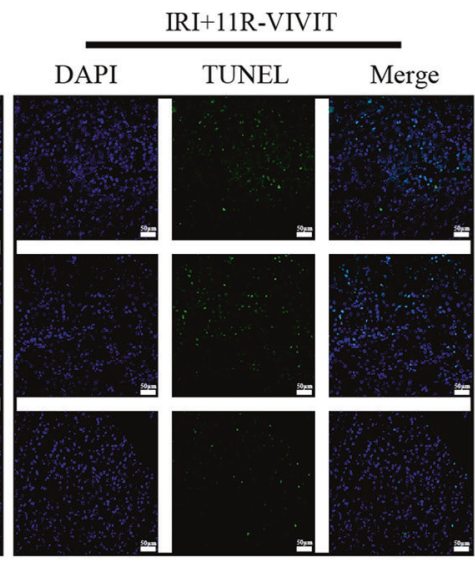

b

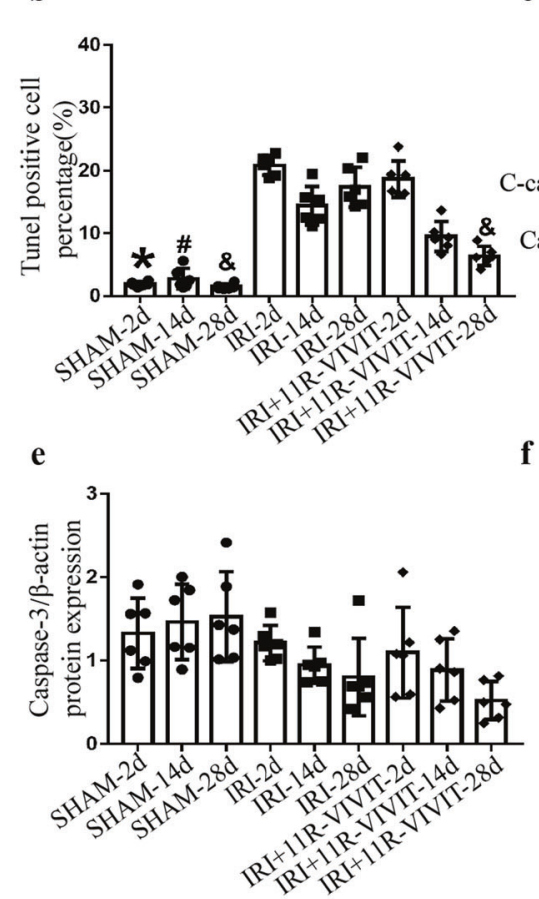

c d
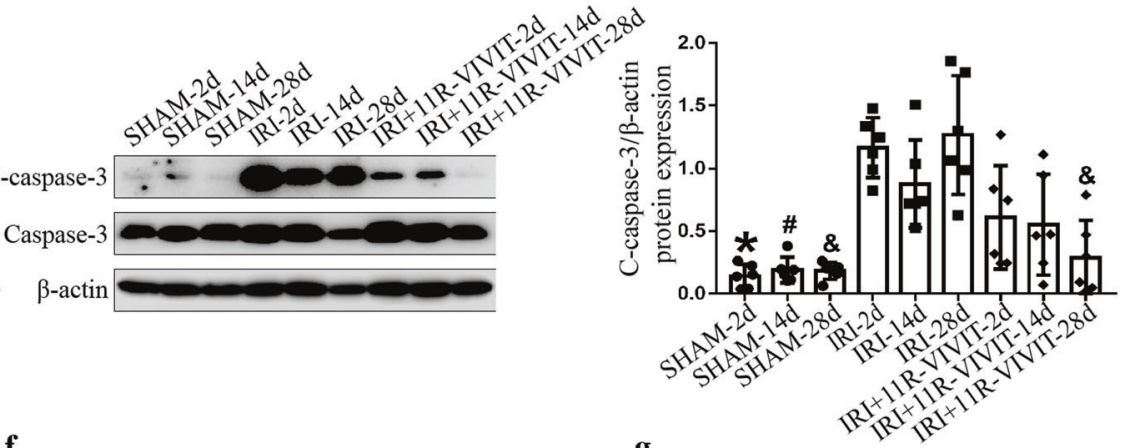

f

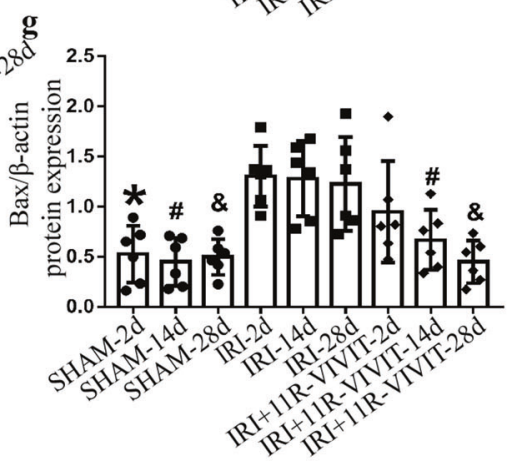

Fig. 6 11R-VIVIT attenuates tubular epithelial cell apoptosis in an animal model of AKI-to-CKD progression. a TUNEL staining of frozen renal tissue sections from the sham-operated, IRI and IRI $+11 \mathrm{R}$-VIVIT treatment groups on the 2nd, 14 th and 28 th day. Scale bars $=50 \mu \mathrm{m}$. b Quantification of TUNEL-positive tubular epithelial cells in the sham-operated, IRI and IRI + 11R-VIVIT treatment groups on the 2nd, 14th and 28th day. c Western blot analysis of the expression of caspase-3 and C-caspase-3 in each group on the 2nd, 14th and 28th day. d-e The quantitative results of C-caspase- 3 and caspase- 3 were normalized to $\beta$-actin. $\mathbf{f}$ Western blot analysis of the expression of Bax in each group on the 2 nd, 14 th and 28 th day. $\mathbf{g}$ The quantitative results of Bax were normalized to $\beta$-actin. ${ }^{*} P<0.05$ vs. IRI-2d, ${ }^{\#} P<0.05$ vs. IRI-14d, ${ }^{\&} P<0.05$ vs. IRI-28d. AKI acute kidney injury, CKD chronic kidney disease, RTECs renal tubular epithelial cells, IRI ischemia-reperfusion injury, C-caspase-3 cleaved-caspase-3.

pathways [23-25]. A previous study [23] showed that the activation of NFAT2 in cardiomyocytes could induce apoptosis through the NFAT2/Fas/FasL signalling pathway. Similarly, activated NFAT2, as a transcription factor, upregulated FasL expression and induced apoptosis in mouse Leydig tumour cells [24]. In addition, another study demonstrated that colorectal cancer cell apoptosis could be induced through the NFAT2/p53 pathway [18], which could activate the mitochondrial-mediated endogenous apoptotic pathway in response to genotoxic and environmental stress. Our previous studies have also shown that NFAT2 mediates high-glucose-induced glomerular podocyte apoptosis through increased Bax expression [15]. As an important mechanism of AKIto-CKD progression, excessive apoptosis in RTECs could lead to inflammatory cell infiltration and cytokine secretion, further promoting renal fibrosis. Inhibiting tubular epithelial cell apoptosis could ameliorate renal fibrosis [9, 10, 25]. In our study, we demonstrated that RTEC apoptosis was significantly increased in an animal model of AKI-to-CKD transition. In vitro experiments also showed that inhibiting NFAT2 could ameliorate the HK-2 cell apoptosis induced by TGF $\beta$. Therefore, we hypothesized that NFAT2 may induce renal fibrosis in AKI-to-CKD progression through the induction of apoptosis in RTECs.

However, one recent study indicated that NFAT2 played a renoprotective role by promoting epithelial regeneration after AKI induced by mercuric chloride, and NFAT2-knockout mice exhibited increased apoptosis, sustained injury, and delayed regeneration [13], which contradicted the proapoptotic role of NFAT2 in renal fibrosis in our study. As different mechanisms are involved in 

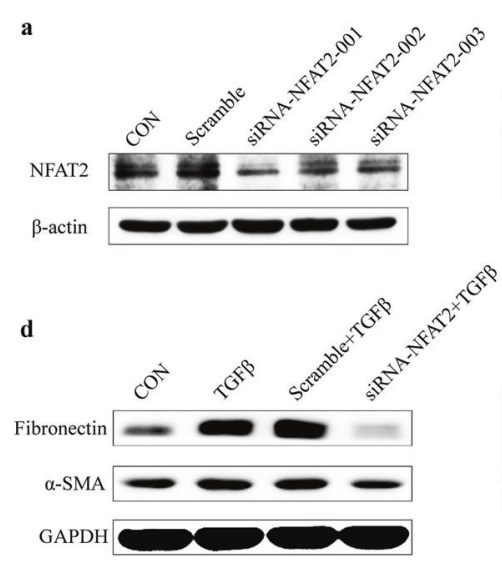

g
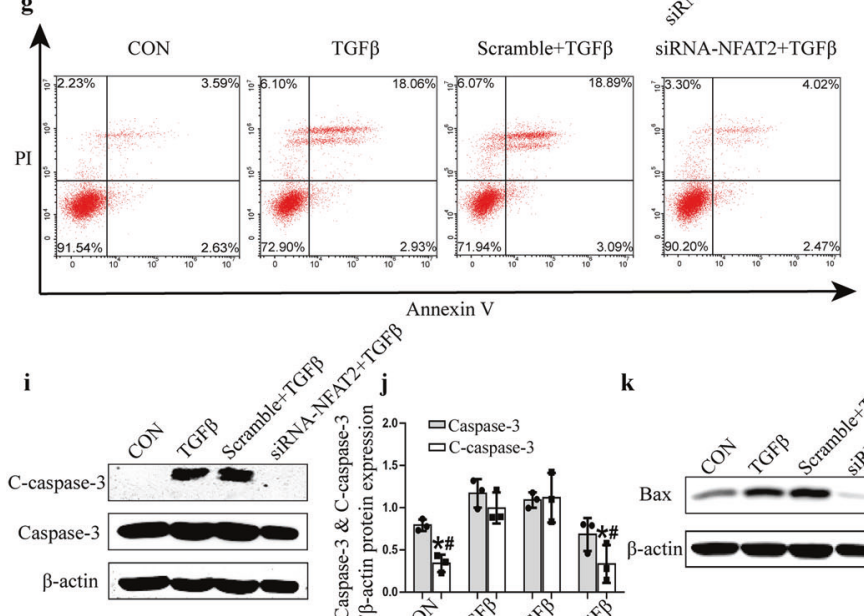

m
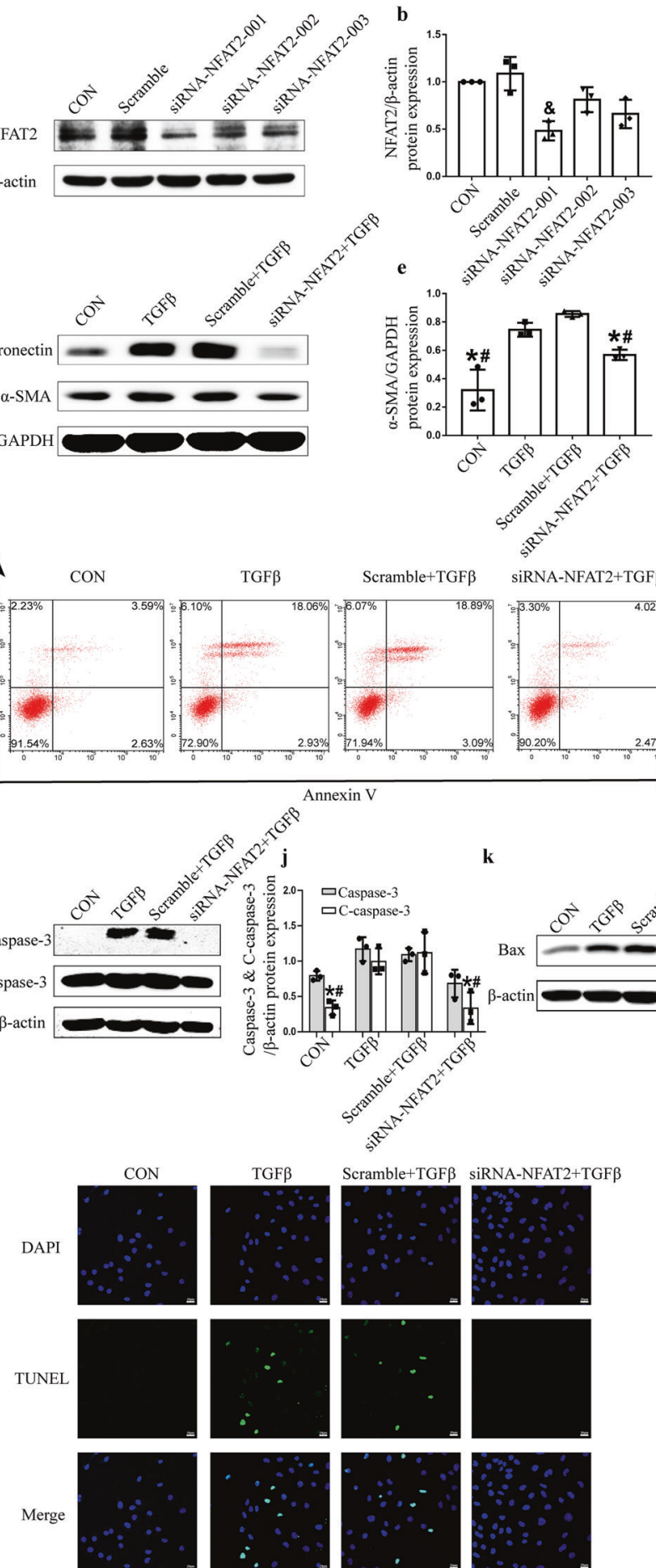

or

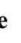

का

की

政

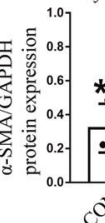

के

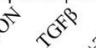
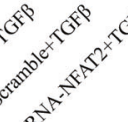

NA-NFAT2+TGFB

$\mathbf{k}$

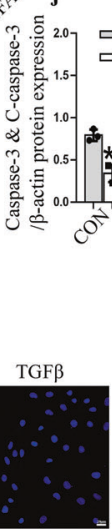

$\square$ Caspase-3
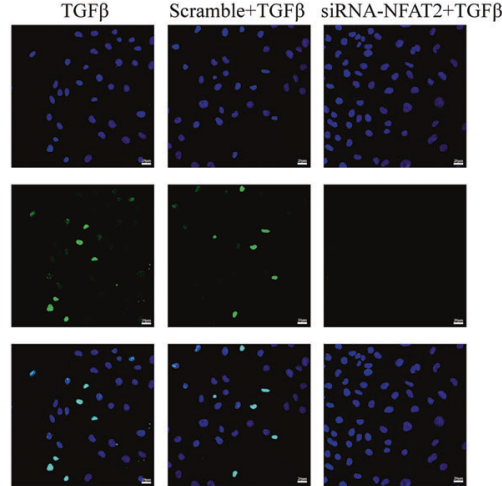

\section{c}
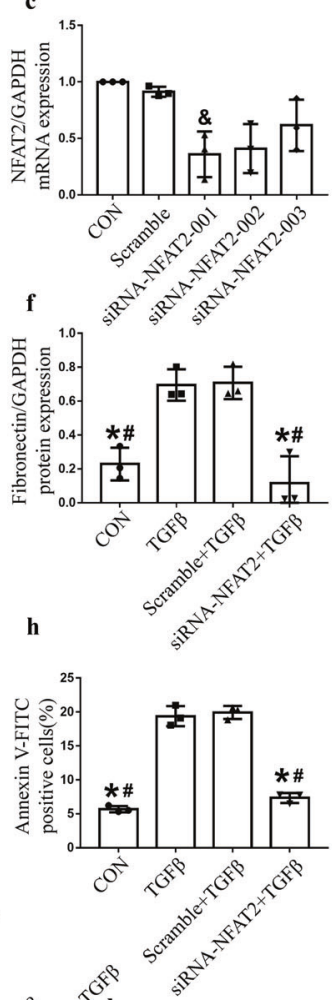<smiles>[CH]1[CH]C1</smiles>
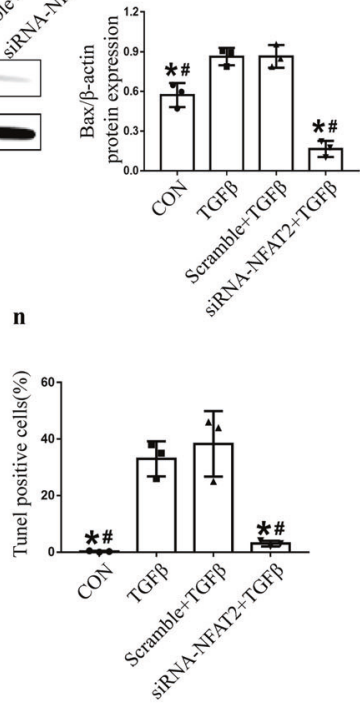

Fig. 7 NFAT2-targeted siRNA attenuates RTEC apoptosis in vitro following TGF $\beta$ stimulation. HK-2 cells in the TGF $\beta$ group were stimulated with $5 \mathrm{ng} / \mathrm{mL}$ TGF $\beta$ for $72 \mathrm{~h}$. The scramble+TGF $\beta$ and siRNA-NFAT2 + TGF $\beta$ groups were transfected with $5 \mathrm{nM}$ scramble or siRNA-NFAT2 respectively, prior to TGF $\beta$ stimulation. a-c NFAT2-targeted siRNA significantly reduced the mRNA and protein levels of NFAT2. Three sequences of the NFAT2-targeted siRNA were used in the present study, and sequence 001 induced significant reductions in the protein and mRNA levels of NFAT2. $\mathbf{d}$ The protein expression of $\alpha$-SMA and fibronectin in HK-2 cells. e-f The quantitative results of $\alpha$-SMA and fibronectin were normalized to GAPDH. $\mathbf{g}$ Apoptosis was examined by flow cytometry. $\mathbf{h}$ Quantification of RTEC apoptosis by flow cytometry. $\mathbf{i}$ Western blot analysis of the expression of caspase-3 and C-caspase- 3 in HK-2 cells. $\mathbf{j}$ The quantitative results of caspase-3 and C-caspase-3 were normalized to $\beta$-actin. $\mathbf{k}$ Western blot analysis of the expression of Bax in HK-2 cells. I The quantitative results of Bax were normalized to $\beta$ actin. $\mathbf{m}$ TUNEL staining of HK-2 cells in the CON, TGF $\beta$, Scramble+TGF $\beta$ and siRNA-NFAT2 + TGF $\beta$ group. $\mathbf{n}$ Quantification of TUNEL-positive RTECs. Scale bars $=20 \mu \mathrm{m}$. ${ }^{\&} P<0.05$ vs. CON and scramble, ${ }^{*} P<0.05$ vs. TGF $\beta$, ${ }^{\#} P<0.05$ vs. scramble + TGF $\beta$. NFAT2 nuclear factor of activated T cells 2, RTECs renal tubular epithelial cells, TGF $\beta$ transforming growth factor beta, CON control, C-caspase-3 cleaved caspase-3. 

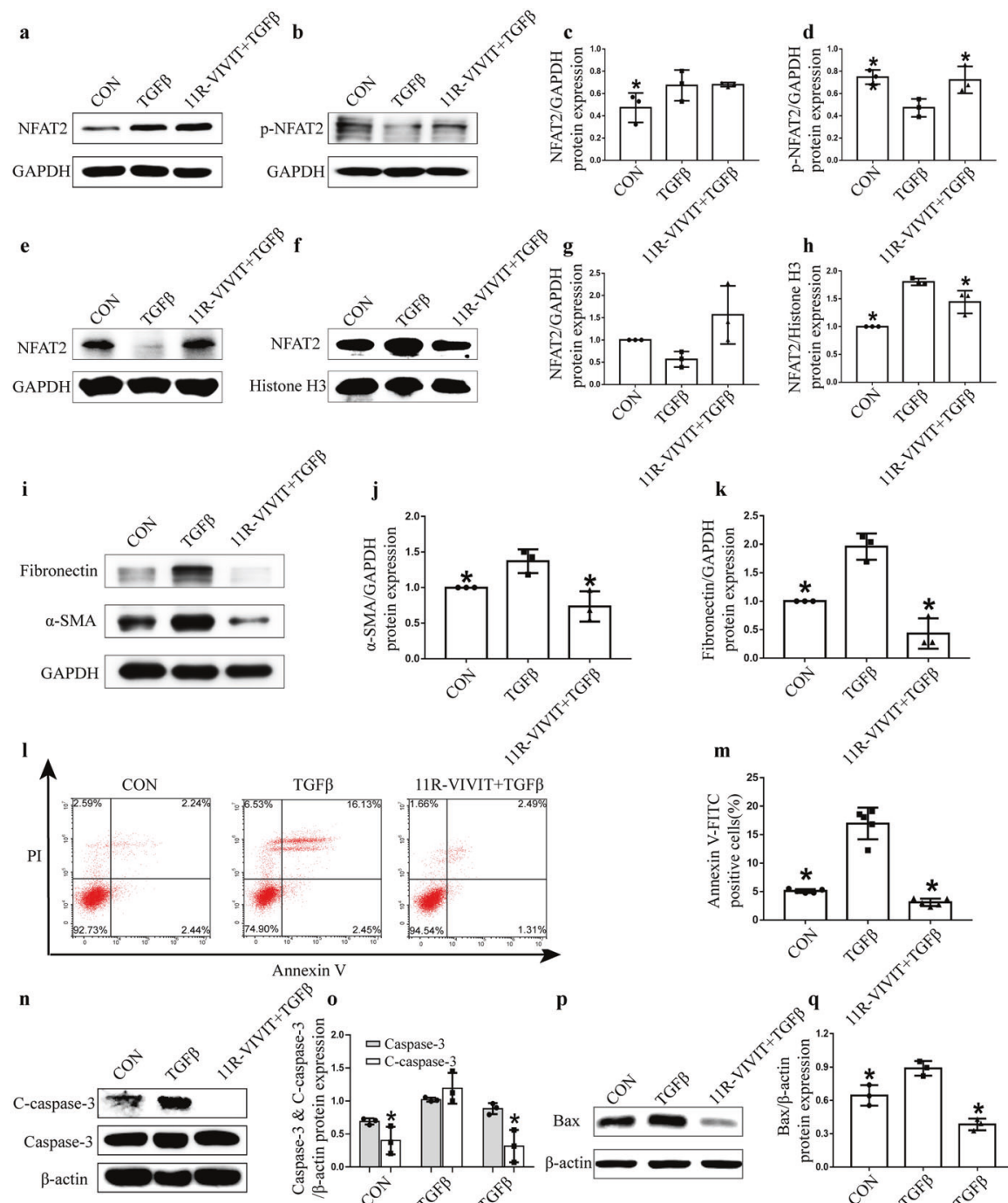

से 0

p

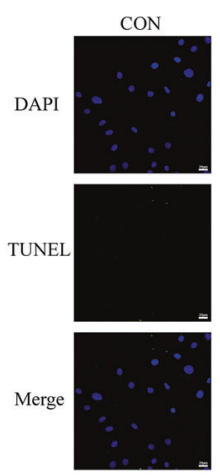

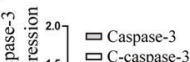

Q
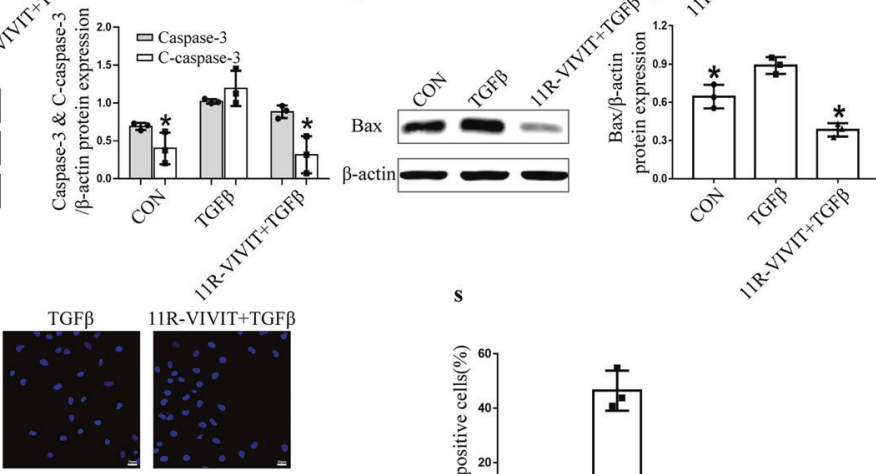

11R-VIVIT+TGF
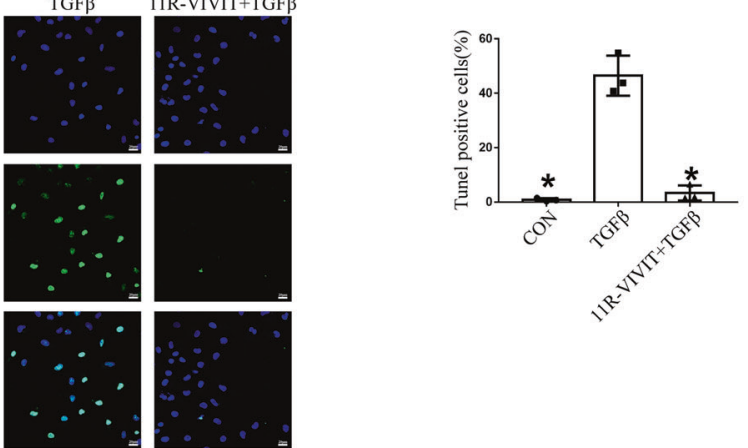

Fig. 8 11R-VIVIT attenuates TGF $\beta$-induced apoptosis in HK-2 cells. a TGF $\beta$ treatment increased total NFAT2 expression in HK-2 cells. b TGF $\beta$ treatment decreased p-NFAT2 (Ser172) expression and 11R-VIVIT could increase the protein expression of p-NFAT2 (Ser172). c Quantification of NFAT2 expression normalized to GAPDH. d Quantification of p-NFAT2 (Ser172) expression normalized to GAPDH. e-f TGF $\beta$ treatment increased nuclear NFAT2 expression in HK-2 cells, and 11R-VIVIT inhibited the nuclear localization of NFAT2. g Quantification of NFAT2 expression in the cytoplasmic fraction HK-2 cells; The results were normalized to GAPDH. h Quantification of NFAT2 expression in the nuclear fraction of HK-2 cells; The results were normalized to Histone H3. $\mathbf{i}$ The protein expression of $\alpha$-SMA and fibronectin in HK-2 cells. $\mathbf{j}-\mathbf{k}$ The quantitative results of $\alpha$-SMA and fibronectin were normalized to GAPDH. I Apoptosis was examined by flow cytometry. $\mathbf{m}$ Quantification of RTEC apoptosis by flow cytometry. $\mathbf{n}$ Western blot analysis of the expression of caspase- 3 and C-caspase-3 in HK-2 cells. $\mathbf{o}$ The quantitative results of caspase-3 and C-caspase- 3 were normalized to $\beta$-actin. $\mathbf{p}$ Western blot analysis of the expression of Bax in HK- 2 cells. $\mathbf{q}$ The quantitative results of Bax were normalized to $\beta$-actin. $\mathbf{r}$ TUNEL staining of HK-2 cells in the CON, TGF $\beta$ and 11R-VIVIT + TGF $\beta$ group. $\mathbf{s}$ Quantification of TUNEL-positive tubular epithelial cells. Scale bars $=20 \mu \mathrm{m}$. ${ }^{*} P<0.05$ vs. TGF $\beta$. TGF $\beta$ transforming growth factor beta, NFAT2 nuclear factor of activated T cells 2 , p-NFAT2 phosphorylated nuclear factor of activated T cells 2, RTECs renal tubular epithelial cells, C-caspase-3 cleaved caspase-3. 
AKI and renal fibrosis, the same signalling pathways or pathogenesis may play different roles in these two processes. For example, autophagy is induced in RTECs during AKI and protects against kidney injury [26]. However, during the AKI-to-CKD transition, the sustained activation of autophagy leads to maladaptive responses and promotes renal fibrosis $[27,28]$. Therefore, inhibiting NFAT2 might result in different effects on RTEC apoptosis in AKI and AKIto-CKD transition. Moreover, it is well known that the mechanisms of kidney injury induced by IRI or toxicants are distinct. In our study, we proved that IR-AKI induced the sustained activation of NFAT2 in RTECs at 28 days. However, in AKI induced by mercuric chloride, NFAT2 expression was significantly increased at Day 3 but returned to baseline levels at Day 5, indicating that different mechanisms associated with NFAT2 are involved in different AKI models. In addition, although NFAT2 attenuation resulted in increased RTEC apoptosis in AKI induced by mercuric chloride, there was no difference in the kidney injury score or Scr levels between wild-type and NFAT2-knockout mice.

Based on the above research results, we hypothesized that inhibiting NFAT2 may be a potential therapeutic strategy for preventing renal fibrosis after AKI. CaN dephosphorylates multiple serine residues near the N-termini of NFAT proteins, enabling them to translocate from the cytoplasm to the nucleus. Traditional CNIs, including cyclosporine and tacrolimus, inhibit $\mathrm{CaN}$ phosphatase activity and further restrain the activation of NFAT [29]. However, the application of CNIs is associated with the progressive loss of renal function, hypertension, neurotoxicity, and an increased risk of malignancy. Intrarenal expression of TGF $\beta$ and other profibrogenic molecules was significantly increased in patients with tacrolimus and cyclosporine nephrotoxicity, which further promoted tubulointerstitial fibrosis [30]. Moreover, other intracellular profibrogenic signalling pathways, including mitogen-activated protein kinase (MAPK) and extracellular signal-regulated kinase (ERK), were also activated by $\mathrm{CNI}$ treatment [31, 32], which restricts the use of CNIs in the AKI-toCKD transition.

$11 \mathrm{R}$-VIVIT is a membrane-permeable NFAT inhibitory peptide composed of several consecutive arginines. 11R-VIVIT was developed based on the conserved CaN docking site within NFAT family members. The 11R-VIVIT peptide interferes selectively with the CaN-NFAT interaction without affecting $\mathrm{CaN}$ phosphatase activity. Compared with CNIs, $11 \mathrm{R}$-VIVIT has a relatively specific inhibitory effect on the nuclear localization of NFAT2 [21]. In our study, we found that 11R-VIVIT could effectively inhibit the nuclear localization of NFAT2 in animal experiments and in vitro. Moreover, our findings showed that $11 \mathrm{R}$-VIVIT could attenuate renal fibrosis and improve renal function after IR-AKI. We also found that $11 R$-VIVIT treatment could effectively attenuate RTEC apoptosis in vitro and in vivo. These data indicated that 11R-VIVIT has a protective effect on the AKI-to-CKD transition. Recent basic research has also revealed its value in tissue repair and the treatment of inflammatory diseases, including fracture healing [33], hearing loss [34], autoimmune encephalomyelitis [35], and cardiovascular disorders [36]. Therefore, as a small peptide, 11RVIVIT has promising future clinical applications.

The possible mechanism of the increased nuclear translocation of NFAT2 in the AKI-to-CKD transition remains unclear. Recent research has indicated that TRPC6 mediates renal fibrosis and that TRPC6 knockout ameliorates kidney fibrosis [37]. Moreover, the inhibition of TRPC6 suppressed NFAT2 nuclear translocation [38]. Therefore, we speculated that the nuclear translocation of NFAT2 may be partly mediated by TRPC6 in the AKI-to-CKD transition.

There are some limitations of this study. We did not specifically attenuate NFAT2 in RTECs in an animal model of AKI-to-CKD transition. In future studies, RTEC-inducible NFAT2-knockout mice will be used to further determine the role of NFAT2 in the AKI-toCKD transition.
In summary, we demonstrated that NFAT2 was activated in renal fibrosis during AKI-to-CKD progression, and the cellpermeable NFAT inhibitor 11R-VIVIT could effectively inhibit NFAT2 activation, reduce RTEC apoptosis, and prevent AKI-toCKD progression.

\section{ACKNOWLEDGEMENTS}

This work was financially supported by National Natural Science Foundation of China (Grant No.81770667), Scientific Research Project of Guangdong Provincial People's Hospital-Summit plan (Grant No. KJ012019120, No. KJ012019433) and GuangdongHong Kong Joint Laboratory on Immunological and Genetic Kidney Diseases (Grant No.2019B121205005).

\section{AUTHOR CONTRIBUTIONS}

$X L L$ designed the study and supervised the entire process. ZYX and WD wrote the manuscript. ZYX, WD, LZ, LF, XCZ, RZL, ZLL and YHC performed the pathological evaluations. ZYX, MJW, ZMX, YHZ, WXS, YH, YY and CLL performed the animal experiments. ZYX, MJW and YHZ performed the in vitro experiments. ZMY, SXL and ZD participated in data analysis. All authors read and approved the final manuscript.

\section{ADDITIONAL INFORMATION}

Supplementary information The online version contains supplementary material available at https://doi.org/10.1038/s41401-021-00833-y.

Competing interests: The authors declare no competing interests.

\section{REFERENCES}

1. Heung M, Steffick DE, Zivin K, Gillespie BW, Banerjee T, Hsu CY, et al. Acute kidney injury recovery pattern and subsequent risk of CKD: an analysis of veterans health administration data. Am J Kidney Dis. 2017;77:742-52.

2. See EJ, Jayasinghe K, Glassford N, Bailey M, Johnson DW, Polkinghorne KR, et al. Long-term risk of adverse outcomes after acute kidney injury: a systematic review and meta-analysis of cohort studies using consensus definitions of exposure. Kidney Int. 2019;95:160-72.

3. Boor P, Ostendorf T, Floege J. Renal fibrosis: novel insights into mechanisms and therapeutic targets. Nat Rev Nephrol. 2010;7:743-57.

4. Chen DQ, Feng YL, Chen L, Liu JR, Wang M, Vaziri ND, et al. Poricoic acid A enhances melatonin inhibition of AKI-to-CKD transition by regulating Gas6/ AxINFkB/Nrf2 axis. Free Radic Biol Med. 2019;134:484-97.

5. Chen DQ, Cao G, Zhao H, Chen L, Yang T, Wang M, et al. Combined melatonin and poricoic acid $A$ inhibits renal fibrosis through modulating the interaction of Smad3 and $\beta$-catenin pathway in AKI-to-CKD continuum. Ther Adv Chronic Dis. 2019;10:2040622319869116.

6. Ferenbach DA, Bonventre JV. Mechanisms of maladaptive repair after AKI leading to accelerated kidney ageing and CKD. Nat Rev Nephrol. 2015;11:274-77.

7. Docherty NG, O'Sullivan OE, Healy DA, Fitzpatrick JM, Watson RW. Evidence that inhibition of tubular cell apoptosis protects against renal damage and development of fibrosis following ureteric obstruction. Am J Physiol Ren Physiol. 2006;290:F4-13.

8. Havasi A, Borkan SC. Apoptosis and acute kidney injury. Kidney Int. 2011;80:29-40.

9. Mao H, Li Z, Zhou Y, Li Z, Zhuang S, An X, et al. HSP72 attenuates renal tubular cell apoptosis and interstitial fibrosis in obstructive nephropathy. Am J Physiol Ren Physiol. 2008;295:F202-14.

10. An JN, Yang SH, Kim YC, Hwang JH, Park JY, Kim DK, et al. Periostin induces kidney fibrosis after acute kidney injury via the p38 MAPK pathway. Am J Physiol Ren Physiol. 2019;316:F426-F437.

11. Macian F. NFAT proteins: key regulators of T-cell development and function. Nat Rev Immunol. 2005;5:472-84.

12. Park YJ, Yoo SA, Kim M, Kim WU. The role of calcium-calcineurin-NFAT signaling pathway in health and autoimmune diseases. Front Immunol. 2020;11:195.

13. Langworthy $M$, Zhou B, de Caestecker M, Moeckel G, Baldwin HS. NFATc1 identifies a population of proximal tubule cell progenitors. J Am Soc Nephrol. 2009;20:311-21.

14. Wang Y, Jarad G, Tripathi P, Pan M, Cunningham J, Martin DR, et al. Activation of NFAT signaling in podocytes causes glomerulosclerosis. J Am Soc Nephrol. 2010;21:1657-66. 
15. Li R, Zhang L, Shi W, Zhang B, Liang X, Liu S, et al. NFAT2 mediates high glucoseinduced glomerular podocyte apoptosis through increased Bax expression. Exp Cell Res. 2013;319:992-1000.

16. Zhang L, Li R, Shi W, Liang X, Liu S, Ye Z, et al. NFAT2 inhibitor ameliorates diabetic nephropathy and podocyte injury in $\mathrm{db} / \mathrm{db}$ mice. $\mathrm{Br} J$ Pharmacol. 2013;170:427-39.

17. Xu S, Shu P, Zou S, Shen X, Qu Y, Zhang Y, et al. NFATc1 is a tumor suppressor in hepatocellular carcinoma and induces tumor cell apoptosis by activating the FasL-mediated extrinsic signaling pathway. Cancer Med. 2018;7:4701-17.

18. Hou N, He X, Yang Y, Fu J, Zhang W, Guo Z, et al. TRPV1 induced apoptosis of colorectal cancer cells by activating calcineurin-NFAT2-p53 signaling pathway. Biomed Res Int. 2019;2019:6712536.

19. Li W, Yu X, Zhu C, Wang Z, Zhao Z, Li Y, et al. Notum attenuates HBV-related liver fibrosis through inhibiting Wnt 5a mediated non-canonical pathways. Biol Res. 2019;52:10.

20. Im J, Kim K, Hergert $P$, Nho RS. Idiopathic pulmonary fibrosis fibroblasts become resistant to Fas ligand-dependent apoptosis via the alteration of decoy receptor 3. J Pathol. 2016;240:25-37.

21. Aramburu J, Yaffe MB, López-Rodríguez C, Cantley LC, Hogan PG, Rao A. Affinitydriven peptide selection of an NFAT inhibitor more selective than cyclosporin A. Science. 1999;285:2129-33.

22. Bechtner G, Fröschl H, Sachse A, Schopohl D, Gärtner R. Induction of apoptosis in porcine thyroid follicles by transforming growth factor beta1 and epidermal growth factor. Biochimie. 1999;81:315-20.

23. Shati AA. Doxorubicin-induces NFAT/Fas/FasL cardiac apoptosis in rats through activation of calcineurin and P38 MAPK and inhibition of mTOR signalling pathways. Clin Exp Pharmacol Physiol. 2020;47:770-7.

24. Chai WR, Chen Y, Wang Q, Gao HB. Mechanism of nuclear factor of activated T-cells mediated FasL expression in corticosterone -treated mouse Leydig tumor cells. BMC Cell Biol. 2008;9:31.

25. Nasu K, Kawakami T, Shinohara A, Sakamoto T, Nangaku M. Munc18-1-interacting protein 3 mitigates renal fibrosis through protection of tubular epithelial cells from apoptosis. Nephrol Dial Transpl. 2020;35:577-87.

26. Kaushal GP, Shah SV. Autophagy in acute kidney injury. Kidney Int 2016;89:779-91.

27. Hernández-Gea V, Ghiassi-Nejad Z, Rozenfeld R, Gordon R, Fiel MI, Yue Z, et al. Autophagy releases lipid that promotes fibrogenesis by activated hepatic stellate cells in mice and in human tissues. Gastroenterology. 2012;142:938-46.

28. Gewin LS. Renal fibrosis: Primacy of the proximal tubule. Matrix Biol. 2018;6869:248-62.

29. Mognol GP, Carneiro FR, Robbs BK, Faget DV, Viola JP. Cell cycle and apoptosis regulation by NFAT transcription factors: new roles for an old player. Cell Death Dis. 2017;7:e2199.
30. Khanna A, Plummer M, Bromberek C, et al. Expression of TGF-beta and fibrogenic genes in transplant recipients with tacrolimus and cyclosporine nephrotoxicity. Kidney Int. 2002;72:2257-73.

31. Wu Q, Wang X, Nepovimova E, Wang Y, Yang H, Kuca K. Mechanism of cyclosporine A nephrotoxicity: oxidative stress, autophagy, and signalings. Food Chem Toxicol. 2018;118:889-907.

32. Wang SC, Tang CHL, Piao HL, Zhu R, Sun $\mathrm{CH}$, Tao $\mathrm{Y}$, et al. Cyclosporine A promotes in vitro migration of human first-trimester trophoblasts via MAPK/ERK1/2mediated NF-KB and $\mathrm{Ca}^{2+}$ /calcineurin/NFAT signaling. Placenta 2013;34:374-80.

33. Hou C, Wang X, Jiang W, Bian Z, Zhu L, Li M. Peptide 11R-VIVIT promotes fracture healing in osteoporotic rats. Int J Mol Med. 2021;48:162.

34. Sekulic-Jablanovic M, Voronkova K, Bodmer D, Petkovic V. Combination of antioxidants and NFAT (nuclear factor of activated T cells) inhibitor protects auditory hair cells from ototoxic insult. J Neurochem. 2020;154:519-29.

35. Lee HG, Kim LK, Choi JM. NFAT-specific inhibition by dNP2-VIVITAmeliorates autoimmune encephalomyelitisby regulation of Th1 and Th17. Mol Ther Methods Clin Dev. 2019;16:32-41.

36. Yu H, van Berkel TJ, Biessen EA. Therapeutic potential of VIVIT, a selective peptide inhibitor of nuclear factor of activated T cells, in cardiovascular disorders. Cardiovasc Drug Rev. 2007;25:175-87.

37. Zhang Y, Yin N, Sun A, Wu Q, Hu W, Hou X, et al. Transient receptor potential channel 6 knockout ameliorates kidney fibrosis by inhibition of epithelialmesenchymal transition. Front Cell Dev Biol. 2021;8:602703.

38. Su Y, Chen Q, Ju Y, Li W, Li W. Palmitate induces human glomerular mesangial cells fibrosis through CD36-mediated transient receptor potential canonical channel 6/nuclear factor of activated T cell 2 activation. Biochim Biophys Acta Mol Cell Biol Lipids. 2020;1865:158793.

\section{(i)}

Open Access This article is licensed under a Creative Commons Attribution 4.0 International License, which permits use, sharing, adaptation, distribution and reproduction in any medium or format, as long as you give appropriate credit to the original author(s) and the source, provide a link to the Creative Commons license, and indicate if changes were made. The images or other third party material in this article are included in the article's Creative Commons license, unless indicated otherwise in a credit line to the material. If material is not included in the article's Creative Commons license and your intended use is not permitted by statutory regulation or exceeds the permitted use, you will need to obtain permission directly from the copyright holder. To view a copy of this license, visit http://creativecommons. org/licenses/by/4.0/.

(c) The Author(s) 2021 